\title{
Drosophila melanogaster and the Future of "Evo-Devo" Biology in Space. Challenges and Problems in the path of an eventual colonization project outside the Earth
}

\author{
By Roberto Marco ${ }^{1}$, David Husson ${ }^{1,2}$, Raul Herranz ${ }^{1}$, Jesús Mateos ${ }^{1}$, and F. Javier
}

Medina $^{3}$

${ }^{1}$ Departamento de Bioquímica de la UAM e Instituto de Investigaciones Biomédicas

“Alberto Sols” UAM-CSIC, C/ Arzobispo Morcillo, 4, Madrid 28029, Spain.

²Centre de Biologie du Développement, UMR 5547 CNRS-Université Paul

Sabatier, 118 route de Narbonne, 31062 Toulouse cedex, France

${ }^{3}$ Centro de Investigaciones Biológicas del CSIC, C/ Velázquez 144, Madrid 28006, Spain.

\begin{abstract}
Space exploration, especially its future phase involving the International Space Station (ISS) makes possible the study of the effects on living systems of long-term expositions to such a strange environment. This phase is being initiated when Biological Sciences are crossing a no-return line into a new territory where the connection between phenotype and genotype may be finally made. We briefly review the paradoxical results obtained in Space experiments performed during the last third of the XX Century. They reveal that simple unicellular systems sense the absence of gravity changing their cytoskeletal organization and the signal transduction pathways, while animal development proceeds unaltered in these conditions, in spite of the fact that these processes are heavily involved in embryogenesis. Longer-term experiments possible in the ISS may solve this apparent contradiction. On the other hand, the current constraints on the scientific use of the ISS makes necessary the development of new hardware and the modification of current techniques to start taking advantage of this extraordinary technological facility. We discuss our advances in this direction using one of the current key biological model systems, Drosophila melanogaster. In addition, the future phase of Space exploration, possibly leading to the exploration and, may be, the colonization of another planet, will provide the means of performing interesting evolutionary experiments, studying how the terrestrial biological systems will change in their long-term adaptation to new, very different environments. In this way, Biological Research in Space may contribute to the advancement of the new Biology, in particular to the branch known as "Evo-Devo". On the other hand, as much as the Space Adventure will continue involving human beings as the main actors in the play, long-term multi-generation experiments using a fast reproducing species, such as Drosophila melanogaster, capable of producing more than 300 generations in 15 years, the useful life foreseen for ISS, will be important. Among other useful information, they will help in detecting the possible changes that a biological species may undergo in such an environment, preventing the uncontrolled occurrence of
\end{abstract}


irreversible deleterious effects with catastrophic consequences on the living beings participating in this endeavour.

\section{Introduction.}

The major problem faced by the rapid biological advances in the years of the transition from the XX to the XXI Century is understanding how the phenotype is actually fabricated from the instructions stored in the genotype and how the environment and additional non-coded physicochemical forces and mechanisms are playing critical roles in the process (Newman and Comper, 1990).

Biological scientists are living in an exciting period. The incredible power of current experimental techniques makes possible to obtain information undreamed a few decades ago. In particular, the explosion of knowledge associated with the completion of the genome project for an increasing number of selected biological species is worth noting. For humans, Drosophila, Cenorhabditis, Arabidopsis and yeast, to name the more relevant eucaryotic organisms, we now have access to the complete genomic sequences, making possible to approach in a new way the problems in understanding how biological organisms function, adapt and evolve. It is true that there are errors in the sequences so far assembled by the industrial-like, mass production sequencing laboratories. It is true that parts of the genome, which present special difficulties and/or seem less valuable, have been left out of the sequencing effort. For example, heterochromatic DNA is not included in the Drosophila genomic sequence. Nevertheless, it is clear that these shortcomings will be eventually corrected in the future. A more formidable obstacle will be to make sense out of the huge amount of data, to identify the functional role of all the genes, old and new, revealed by this sequencing effort. Biological research is equipped now with very powerful research tools to approach this task and accomplish, at least in theory, but extremely costly in terms of manpower and economic research support. It is therefore critical to increase the resolution and to overcome the limitations of present day Biology, still, in our view, a descriptive, qualitative discipline. Biology has began to develop new methods to analyze and integrate the information on the behavior of large networks of elements that we increasingly recognize as being underneath the function of biological systems and their adaptations to their environments, life styles and survival rates.

An important area of Research that we believe could contribute to this new phase of investigation can be linked to the exploration and the mastery of Space, to the challenge brought about by the objective of sending and maintaining living systems outside the limits of our Biosphere (Moody and Golden, 2000).

Biological Space Research has been pursued during the last decades with increasingly sophisticated means. Nevertheless, so far only relatively short-term experiments have been possible. Even in the Russian MIR Station, biological experiments have been operated usually during a few weeks. Exception has to be taken of the few human beings that have been exposed there during longer periods of time, periods not so 
long in relative terms if we take into account the human life-span. Another exception could be found in the uncontrolled specimens, collateral to these experiments, such as the microbiological flora that colonized and evolved in the Station during its years of operation, but which has not been studied in this connection or this knowledge made available to the scientific community.

The next phase of the Space Exploration will allow the performance of much longer biological experiments in Space. For example, once the International Space Station is completed, controlled long-term experiments using key biological model systems will be for the first time possible. It is true that financial limitations are putting enormous constraints on this possibility. The crew number has been reduced for safety reasons to a number that makes the availability of crew time one of the more precious resources in the ISS. The original dream of the scientists involved in Space Research was that laboratories completely equivalent to those that we use in our Ground Institutes would be installed in the ISS modules. At least for the next few years, the scientific community interested in using the ISS will have to cope with these limitations. This means that experiments have to be run using mostly automatized Hardware and postponing the more work intensive analysis until samples are recovered on the Ground, something that will only occur at certain times, taking advantage of the periodic refurbishing flights to the ISS. Thus, it will be critical to advance in the improvement of methods involved in the preservation of samples, both living and fixed, as well as samples maintained in ways that will allow the recovery of critical molecules (DNA, RNA, proteins) to run specific genomic and proteomic experiments on them after these periods of time. The availability of microarray methodology is an exciting development linked to the Genome project, making possible the investigation of the overall response of the genome of key biological model systems as they experience and adapt to different situations.

In fact, in this Chapter we will discuss the objectives and the problems behind our purpose of establishing a permanent colony of Drosophila in Space to study how a highly organized multicellular organism will respond and adapt to this foreign environment.

Furthermore, the phenotypic and genotypic changes actually observed in these long-term experiments will provide useful information on the ways current earth-based biological systems could adapt to unusual environments, probably providing clues on how evolution could have proceeded in the past in our planet. Another complementary line of research is founded on the efforts to build and control self-sustaining life support systems that will probably be the initial scenarios in which the problems behind the project of the colonization outside our Planet will be identified and tested.

In addition, even if relatively little could be learned about evolution of new adaptations, the exposure during long periods of time and many generations of selected model systems will be a necessary step forward to establish the conditions for the future colonization of another planet, especially if human beings are going to participate in this undertaking. This precaution would help unveiling any unforeseen problems before they affect the persons participating in the enterprise. 


\section{The earlier evidence. The earliest phase (1961-2001).}

What do we know about the exposure of living systems to the Space environment and to the possible adaptations to the Terrestrial environment that may be affected in long-term exposures to an alien environment? We will briefly summarize in the following paragraphs what we think we know, bringing into focus some paradoxes that seem to have emerged from this work. The dates indicated above cover the period between the first Space flight in which an organism was recovered alive (Yuri Gagarin possibly accompanied by a few fruitflies in his pockets) and the disappearance of the Russian MIR Station.

\section{A) Effects on Animal Development}

Although Biological Research in Space has been limited by many constraints, during the past fifty years this type of experimentation has been steadily pursued by laboratories in USA, the former Soviet Union, Japan and Europe (see Claassen and Spooner, 1994, for a review). As a consequence, a certain body of knowledge on the response of animal organisms, tissues and cells to the Space Environment has been accumulating. In fact, the starting point for our argument is the following apparent paradox. While most cells and tissues appear to sense and change their behavior in response to Microgravity, whole animal organisms have developed normally in spite of the clear involvement in the developmental process of the reported affected cellular processes and components. The paradox is made more forceful by the realization that gravity is a weak physical force and should be hardly effective on tiny microscopic objects, such as cells in the absence of specialized organelles of high density like those existing in plant systems (Albrecht-Buhler, 1990, Mesland, 1992). In spite of this fact, a relatively large number of experiments indicate that the smaller, simpler systems made up by isolated cells and/or tissues feel the absence of the normal gravity vector. As a consequence, they change many of their basic intrinsic processes, such as cell proliferation, cytoskeletal organization, signal transduction pathways, particularly those involving $\mathrm{G}$ proteins and protein kinase $\mathrm{C}$ action, etc (for reviews, Claassen and Spooner, 1994, Moore and Cogoli, 1996, Cogoli and Cogoli-Greuter, 1997). Examples of these responses are: diverse ciliated and flagellated protozoa (Hemmersbach and Häder, 1999), lymphocytes, monocytes and macrophages (Cogoli et al, 1993, Cogoli 1993, Chapes et al, 1994, Schmitt et al, 1996, Cogoli-Greuter et al, 1996, Lewis et al 1998, Lewis et al, 2001), epidermoid carcinoma cells (de Groot et al, 1990, 1991), connective tissue cell lines (Chapes et al, 1994), bone cells (Bikle et al, 1994, Van Loon et al, 1995, Vico et al, 1998, Burger, Klein-Nulend, 1998), muscle and nerve cells (Gruener and Hoeger, 1990, Reitstetter, 1994, Gruener et al, 1994), Drosophila Schneider S-1 cells (Schatten et al, 2001), etc. In conclusion, simple, isolated cell systems, in principle too small to be evident targets of the weak force of gravity, show in general a clear response when exposed to a Microgravity environment. They sense the absence of gravity and change important cellular responses such as signal transduction pathways and the cytoskeletal organization. Equivalent changes have been reported for experiments using simulated Microgravity (Walther et al 1998, Clejan et al, 2001, Uva et al, 2002, Grimm et al 2002) 
On the other hand and in spite of the extensive involvement of these processes (Signal transduction pathways and CSK organization) in Development, the larger and more complex multicellular organisms seem more robust, showing completely normal morphologies and functions, even when their development has occurred totally in Microgravity. This result has been verified in a wide variety of model systems organisms (nematodes, Drosophila, sea urchins, fishes and amphibians, etc). from low to highly evolved invertebrates to vertebrates (see Marco et al, 1996 for a review). Occasional reports of developmental alterations have not been reproduced in posterior, better conducted and controlled experiments. On the other hand, in other experiments, transient modifications in early developmental events have been observed, modifications that eventually are rescued. There are hints that some developmental processes may be affected (for example, in amphibians), but the formidable regulatory capabilities shown by developmental systems, are able to correct and overcome in the long run any alterations that Microgravity conditions introduce in the normal course of animal development (Souza et al, 1995, Husson, 1998, Duprat et al, 1998). We have little understanding of the correcting processes. Therefore, complete normal development has been the usual outcome of these experiments. It has to be pointed out that development, $i$. $e$., the production of complex highly differentiated organisms, begins with a single huge cell, the embryonic zygote. This make these results even more paradoxical since larger size should make the earlier embryonic cells much better targets for the weak force of gravity. Not many of these experiments have been able to study the cellular events themselves during development due to the problems involved in the successful handling of these complex organisms during the Space flights. In some cases, morphological details of some intermediate structures during development suggest abnormalities in cell proliferation during development, such as the finding of a thicker blastocoel roof during early Xenopus laevis development in Microgravity (Souza et al, 1995, Ubbels et al, personal communication). Similar results have been obtained by French investigators using a different amphibian species, Pleurodeles waltl (Husson, 1998). Different anomalies were detected in the early phases of development (cleavage and neurulation) but the majority of the embryos achieved a normal differentiation and morphogenesis. In general, the reported changes were fully compatible with normal development. This is not an unusual result in Developmental Biology where the robustness of the systems has been traditionally ascribed to the so-called regulatory behavior at certain stages of embryos and parts of embryos. In relation to this regulatory behavior, there is the question of the redundancy of many elements that build the regulatory networks and pathways controlling development (Malacinski, 1990, Li \& Noll, 1994, Cooke et al, 1997, Tautz, 2000). This redundancy is especially remarkable in certain processes and pathways such as those involved in cytoskeleton organization and signal transduction, precisely those more often reported to be affected by exposure of isolated cells to Microgravity.

In several cases, alterations in functional properties of the organisms linked to the strong reduction in gravity forces have been described. In plants, geotropic response is impaired and in mammals, loss of bone tissue and changes in functioning correlated with switches in the specific proteins isoforms expressed in these muscles have been reported (Kraemer et al, 2000). In sea urchins, effects on the mineralization under exposure to Microgravity have been described, although it is not yet clear if they are direct or indirect 
(Marthy et al, 1998). Effects on mitochondria and cilia in sea urchin embryos have also been reported (Schatten et al, 1998).

Our group has been involved in the study of this problem since almost two decades ago using mainly as the experimental system, Drosophila melanogaster, a small and resilient arthropod, probably the higher animal organism better known from a developmental point of view. Drosophila has been one of the organisms more often used in the earlier history of Space Research, both in Russian and American Space Flights (for reviews, see Miquel, 1984, Miquel and Souza, 1991). These earlier experiments established the compatibility of this small organism with the constrains and stresses of Space Travel, showing that it was possible to send and recover many developed fruit flies that had underwent development under Microgravity conditions. The logical constraints in these earlier flights made difficult to apply strict quantitative analyses to the recoveries and properties of these organisms, leaving open the possibility that developmental effects had remained undetected or were eventually rescued during the operation. Our initial experiments carried out in the middle 80's and early 90's (Marco et al, 1986, Vernós et al, 1989, Marco et al, 1992) seemed to support some of these expectations. More recent experiments (see Marco et al, 1996) carried out with better hardware allowing a better oxygenation of the samples have clarified these issues, showing that development of Drosophila in Microgravity is quantitatively and qualitatively normal, at least when one looks to the final product, the developed larva and adult fruit fly. Large quantities of larval and adult animals in the order of thousands were recovered from these experiments (Table 1). The detailed analysis of the results failed to verify the earlier found $50 \%$ reduction in the number of normal developed flies in Microgravity, pointing even more clearly to the paradox posed by the results obtained with simpler unicellular systems. Furthermore, the enlarging list of organisms that have been sent into Space and whose development in Microgravity has been studied, indicates the generality of this conclusion. Thus, the complex process of development integrating all cellular processes, such as cell proliferation and movements, cell communication and signal transduction, cellular, extracellular and tissular differentiation, appears to be normal in Space, while the same processes studied in many isolated cell systems seem to be altered.

Table 1.

Results of IML-2 Development Experiment

Drosophila larvae/flies developed from eggs laid by flies at the end of the third day of a Shuttle Flight of 18 days after the first embryo collection had been removed

\begin{tabular}{|l|c|c|c|c|c|}
\hline \multirow{2}{*}{$\begin{array}{l}\text { Number of developed } \\
\text { animals recovered }\end{array}$} & \multicolumn{2}{|c|}{ Flight Containers } & \multicolumn{2}{c|}{ Simultaneous Ground Control } & Trays* \\
\cline { 2 - 6 } & Static (0g) & Centrifuge (1g) & Static (1 g) & Centrifuge (1.4g) & \\
\hline Frozen & 2155 & 1875 & 1822 & 2028 & 7 \\
\hline Alive & 719 & 947 & 554 & 558 & 4 \\
\hline Total & 2874 & 2823 & 2376 & 2586 & 11 \\
\hline
\end{tabular}

* The number of trays recovered from the containers (two for each experimental condition) with 40 female and 10 male flies each 


\section{B) Aging and Behavior}

One of the main challenges in current Biological Research is to understand the aging process in complex multicellular organisms (Partridge and Barton, 1993, Shigenaga et al, 1994, Finch and Tanzi, 1997, Johnson et al, 1999). The similarities of the physiological changes and adaptations to Microgravity endured by astronauts with the aging response in humans have been repeatedly emphasized (Wang, 1999, Burger and Klein-Nulend, 1999). Although a species life span most probably is an evolutionary acquired trait (Rose, 1991), it is still unclear how much of it is genetically or epigenetically modifiable. Insects, mostly composed by post-mitotic cells with relatively short life spans are a group of organisms where some features of the aging response can be optimally studied. The idea that an enhanced metabolism, the so-called rate-of-living theory, accelerates the rate of aging has been one of the explanations of the phenomenon, earlier advanced by scientists (Pearl, 1928). The idea was further refined (Miquel and Fleming, 1984, 1986) linking the aging phenomenon to the increase in mitochondrial function, consequence of the increase in metabolism (Sohal and Weindruch, 1996, Wallace, 1999). Environmental epigenetic treatments such as changes in the temperature of rearing are known to modify the aging response in Drosophila (Miquel et al, 1976, Lints and Soliman, 1988, Rose, 1991). Measurement of the oxygen consumption at different temperatures provided evidence in agreement with the mitochondrial involvement in Drosophila aging (Miquel et al, 1976). Using Musca domestica, the common housefly, several environmental treatments, including the level of physical activity, have been shown to modify the rate of aging (Agarwal and Sohal, 1994). Exposure to the Microgravity environment of Drosophila male imagoes was another epigenetic treatment influencing the aging process in Drosophila, as previously reported by our groups (Miquel and Philpott, 1978, Marco et al, 1986). In these experiments, exposure to the space environment during one to three weeks as young imagoes resulted in a shortening of life-span after recovery of the animals from space, when compared to the ground control animals measured in parallel in the same conditions.

An idea that could unify these results was that exposure to Microgravity resulted in behavioral changes, such as an increased locomotor activity. The consequent enhanced mitochondrial respiration of the flies may accelerate aging, as has been shown for raised temperatures, hyperoxia or high physical activity on the ground (Miquel et al, 1980, Agarwal and Sohal, 1994). Moreover, the idea that the free radicals produced in metabolism could be related to the aging process has received support from experiments carried out in Drosophila. An artificially induced increased genetic dosage of two enzymes, copper-zinc superoxide dismutase and catalase, involved in the removal of deleterious free radicals, oxidative products of mitochondrial metabolism, resulted in the slowing down of senescence (Orr and Sohal, 1994). The hypothesis that a progressive alteration in mitochondrial activity is central to the decrease in functional performance linked to the aging process in high organisms obtained direct experimental evidence from our laboratory. We showed that in Drosophila this decrease in mitochondrial function is linked not to a modification in the quantity and/or quality of the mitochondrial DNA but rather caused by a general and significant decrease in the levels of RNAs coding for mitochondrial components (Calleja et al, 1993). These results have been later confirmed in other laboratories (Schwarze et al, $1998 \mathrm{a}$ and b). 
As already mentioned, earlier Space experiments had indicated that young male Drosophila flies exposed to microgravity showed an acceleration in aging. In a 14.5 days Space Shuttle Flight (IML-2), we sent 300 young male flies with the purpose of confirming these findings. We could establish that changes in fly behavior were responsible of the effect in accordance with the proposal that alterations in mitochondrial metabolism may be involved in the aging response. Through repeatedly video recording, we found a very marked increase in the fruit-flies locomotor activity in Space (Fig. 1). The males showed accordingly an accelerated aging response upon recovery, both in terms of physiological vitality assays (mating and negative gravitaxis) and of life-span curves (Benguría et al, 1996). Recently, an American team has been able to verify that the increase in motility is observable quite rapidly, even in short microgravity exposures, such as those achieved in Sounding rockets or in parabollic flights (Miller and Keller, 2000).

The involvement of mitochondrial metabolism is also suggested by the finding of a greater decrease in mitochondrial 16S ribosomal RNA in Microgravity exposed flies than in ground controls. On the other hand, a parallel 1xg-centrifuge control did not show such differences in the life-span curves when compared to flies exposed to a similar centrifugation on the Ground. Drosophila females also increased their locomotor activity but did not show differential changes in the life-span curves, although they showed some initial signs of increased aging.

Another point is why the Drosophila imagoes increase their locomotor activity in Microgravity. Although we do not know the reason, we suspect that this behavioral response of increased motility may be related to the immaturity of the young flies used in the experiments and the negative gravitaxis escape response shown by Drosophila on the Ground (Miquel et al, 1972). Young imagoes may have a more immature behavioral response and therefore, they may overreact to the absence of a normal reference gravity vector, resulting in a continuous crawling in search for a "higher" site in the container that obviously cannot be found in Microgravity. Earlier results indicate that mature older flies (two or more weeks old) exposed to Microgravity did not show the acceleration in the aging response that the young flies experience (Miquel and Philpott, 1978). More recent and unpublished work from our laboratories (the Bion-10 satellite flight) confirmed this difference for the more mature flies exposed to Microgravity (de Juan and Marco, unpublished). Interestingly, since older animals exposed to all the circumstances associated with a Space flight showed less acceleration in the aging response, nor such an increase in locomotor activity in space, strongly reinforces the conclusions of the IML-2 experiment and the connection between Drosophila metabolic rate and senescence. In this case, the fully mature older imagoes may use additional cues and, thus, avoid or show a less marked behavioral response. A testable prediction would be that Drosophila mutants affected in their negative gravitaxis response may show a change in the Microgravity response or an increase in the locomotor activity on the Ground, depending on which phenotype they show. Drosophila strains with altered response to the gravity vector on the Ground have been repeatedly selected in the past (Hirsch and Ksander, 1969, Dobzhansky et al, 1972) and in our parallel Ground effort, we will reproduce and study the selection of similar gravitaxically altered strains. Finally, the long term cultivation of Drosophila flies 
in abnormal gravitational conditions and the consequent production of multiple generations will allow to test if there is a change in behavior as the culture become established and a corresponding mitigation of the effects on the life spans of the flies. Information on how insects do sense the gravity level is becoming available (Horn and Foller, 1998, Texada et al, 2002) and the physiological basis of the altered response in Drosophila may be linked to changes in the sensing or in the responding mechanisms.

\section{C) Our current goals in Biological Space Research.}

As already described, there is evidence that multicellular organisms as well as simple unicellular organisms are able to sense the absence of gravity and modify their functional, cellular and molecular properties in ways that suggest the possibility of adaptations to the Space Environment. Obviously, a more thorough study of this possibility will have to wait until we have access to the capability of performing longterm experiments in the Space Environment, something feasible with the facilities that will become available once the International Space Station becomes fully operational. Therefore, it is worthwhile to concentrate now in the preparation of the experimental setups that will be able to take advantage of this unique research environment.

Our objective in this enterprise will be to use the possibilities associated to one of the current more popular research systems, Drosophila melanogaster, to gather new data that helps in solving one of the more important questions in Biology, how the instructions contained in the genotype are transformed into particular phenotypes under the influence of the environment (Fig. 2). Current existing biological systems are buffered against the alterations of the Environment. In fact, they tend to be relatively insensitive to big changes in environmental conditions, explaining why there has been a general failure in detecting developmental effects in Microgravity, as mentioned above. On the other hand, we can easily perceive that living systems are well adapted to the environmental conditions in which they actually live. In addition, we are also well aware that biological systems, such as Drosophila, easily respond to artificial selection protocols producing animals that have altered morphological or physiological features that are prompted by the selection protocols (Pigliucci, 2001). Furthermore the current explosion in basic knowledge in these systems makes for the first time feasible to try to clarify how the selection protocols work.

\section{The current phase. Preparation and Use of the ISS}

\section{Potential impact of the Space Exploration Program in the field of Biological Evolution}

The current phase in the Space Exploration Program is the building and putting into operation of the International Space Station. It can be argued that the ISS is not the final goal of the Space Exploration Program itself. It may be just a milestone in a more ambitious long-term Project, the transformation of the environmental conditions and the 
colonization of other Planets and Celestial Bodies by earth-originated living systems. In any case, if the presence of man in Space is going to be more than episodic, it is important to initiate the investigation of the long-terms effects of the exposure of living organisms to completely different environments outside the Earth. The use of appropriate model systems is mandatory, among other reasons, to prevent that man itself serves as the guinea pig for these long-term experiments. Thus, the establishment of more or less permanent and autonomous (self-sustaining) colonies of living beings in Space should be given priority in the Space Programs. In addition to testing and solving the practical problems behind this Enterprise, an additional question that we may ask is how the living systems will respond to this challenge? Although many of the details are still unclear, very likely life has appeared and evolved on Earth by relative gradual mechanisms and processes known as Biological Evolution. Once biological systems of a certain complexity appeared, systems endowed of the main basic properties of cells (genetic information, catalytic activities and membranes), neo-Darwinian mechanisms of Evolution could start to operate and, eventually, the early sets of pre-biotic systems were replaced by more complex and autonomous organisms. The immense diversification of Living Systems currently found in our Planet has been achieved as a consequence of dialectical tensions operating on and in living systems, i. e., the maintenance of short-term "exact" replication together with long-term variation, selection and adaptation to environmental conditions. In fact, the importance of getting a better understanding of Biological Adaptation, including the solution to the long standing controversy on the relative importance between neutral and selective forces in the shaping of this diversity is being increasingly discussed and studied (Goodwin, 1990, Rose and Lauder, 1996, Raff, 1996, Gerhart and Kirschner, 1997, Marco et al 1999, Arthur, 2000, Brock, 2000).

Although the increase in our knowledge of the actual molecular, cell, organism and population related mechanisms involved in these processes is remarkable and continues increasing at an accelerated path, most questions remain unanswered. Some are quite difficult to study, in part because the process is quite slow, the evidence insufficient to discriminate among powerful theoretical alternatives and the results many times unexpected even under laboratory conditions (Rose and Lauder, 1996). It is not even clear what makes an environment radically new and different and how the response is influenced by random events.

One interesting consequence of performing relatively modest and feasible experiments using the ground and flight facilities that the new phase of the Space Exploration will be offering to the Scientific Community is that it will contribute to the answer of these questions in addition to the identification of any potential deleterious effect on living beings of the long-term exposure to the Space environment. This is an important goal of the group of experiments discussed in this Chapter. A shorter-term goal is the development of the hardware and techniques that will make possible the establishment of permanent colonies of certain Model Organisms in Space. Obviously, we intend to take advantage of all the current powerful techniques available to examine the modifications that the organisms will experience during this long-term exposure to this unusual environment. In our case, using Drosophila, we will monitor the phenotypic and genotypic changes that they will undergo as well as to identify the particular molecular and cellular mechanisms that will be modified in this process. This type of 
experimentation should follow equivalent ground experimentation in modified environments, using similar facilities and techniques as will be available later in the ISS. In these ground experiments, we will also monitor the overall changes that Drosophila may undergo when permanently exposed to modified gravity environments on the Ground or selected for different responses to the gravity vector.

The use of Ground Simulation Facilities is a key part of the present phase of Research. It is true that gravity is a pervasive force and it is impossible to really eliminate its action when standing in our Planet. Nevertheless, a growing arsenal of techniques and equipment is becoming available in ground research laboratories, particularly in special Centers supported by Space Agencies, such as the User Support Operation Centers that the European Space Agency is fostering in Europe. This approach allows at least three things, 1) to test the hardware and technical support of Space Experiments much before they are submitted to the real conditions in Sounding Rockets or in parabolic flights. 2) to test the feasibility of the actual experiments selected for Space Missions and 3) and more importantly in a first approximation, to select from the enormous list of possible experiments, animal strains, gene markers, etc, those that more probably could give information in real Microgravity and Space conditions. Among the types of equipment currently available in these Centers the following ones seem the better adapted for the Drosophila experiments. 1) Compensating gravitation equipment such as clinostats, where sample orientation is regularly changed with respect to the normal gravity vector. Originally the equipment consisted of rotating clinostats (slow and fast) that could not easily accommodate large samples and pieces of hardware and observation equipment such as microscopes and/or video cameras. The recent availability of the 3D clinostat also called the Random Position Machine has solved many of these problems. 2) the Magnetic Levitation Machine is based on the magnetic susceptibility of the individual atoms that make up the molecules when subjected to magnetic fields of enough intensity (Berry and Geim, 1997). The size of the exposed organisms is limited in the available equipment, but it can easily accommodate Drosophila samples. On the other hand, it may not be used for long-term exposures. 3) Hypergravity delivering centrifuges in the range of a few g-level and capable of accommodating observation equipment are also available.

In this respect, it has to be pointed out that applying centrifugal forces, $i$. e., hypergravity conditions, to developmental systems was an often used experimental tool in the hands of certain classical developmental biologists, leading to important phenotypic effects on the development of certain biological species (Kalthoff, 1996). On the other hand, there is hardly any information on the effects of the exposure of Drosophila during development to Ground altered gravity simulation facilities.

Finally, we believe that the selection of the key model organisms that could be incorporated in experiments of long-term exposure to the Space Environment is very important. They could become part of the Life Support Systems that will allow the participation of Human beings in this new phase of Space Exploration. Furthermore and in relation to the problem of how evolutionary adaptation of living beings actually occurs, it is important to study the adaptation of these organisms to extraterrestrial environments. As stated by Edgerton and Roy in a relatively recent review (2000) “ it is not really a 
question of whether humans have the resolve to explore space. The question is which generation will be able to take advantage of this resolve”.

\section{The reasons to select Drosophila for long-term experiments in Space.}

As already mentioned, Drosophila is a relatively small resilient organism, which has been used quite often in Space Research in the past. It is possible to grow relatively large quantities of flies in relatively small containers (but see below for some open questions). We are setting experimental conditions that will allow to obtain as many generations as desired (or as the storage capability and the occupancy of the International Space Station will make possible) to monitor the long-term effects of the exposure to the Space Environment of a population of a complex multicellular organism. Since the life cycle of Drosophila takes approximately two weeks at room temperature, this means that per year we will be able to grow more than twenty generations in Microgravity. Furthermore, since Drosophila is the higher organism for which we know more in terms of genetics and development (Powell, 1997), it will be possible to study what genetic changes do occur in this environment and how they can explain the phenotypic changes, biochemical, morphological, functional and behavioral that may be occurring during this long-term experiment.

For example, as already mentioned, the complete euchromatic sequence of Drosophila is now accessible, completed by a joint effort from private and public sources. The current impression is that the sequence is very useful, although it will be corrected in the future to become completely operative. Nevertheless, the availability of the sequence and the corresponding identification of the genes included in the sequence is providing tools for the examination of the gene expression profile in the fly when exposed to Microgravity and as it adapts to the Space environment. The usefulness of this approach is indicated in many publications, where the applications of microarrays to the detection of the changes in whole group of genes have been studied. In particular, the differential expression of the one-third of the genome complement during Drosophila development has began to be explored (Arbeitman et al, 2002). They show data generated at multiple time points throughout Drosophila melanogaster development, and identify new genes engaged in a broad spectrum of processes, including the patterning of the early embryo and senescence in adults. There are already publications that indicate the potentiality of this approach in detecting the overall changes of gene expression in Space (Hammond et al, 1999, Lewis et al, 1998, 2001) or in simulated Microgravity on the Ground (Khaoustov et al, 2001). Many genes change expression when compared to the ground controls confirming what we have already mentioned, $i$. .e, that isolated cells sense the absence of gravity and change their responses. What is still left to know are the functional consequences and the adaptations produced by these changes in response. During a long period of time, classical genetics have been merging with molecular genetics, but now this confluence is being extended to quantitative genetics. The recent advances in Genomics have defined the number of genes in a series of familiar organisms. Even though these numbers may change as information on Functional Genomics becomes available and as information on different strains in the same species is obtained, they put a limit on the number of ways that this information can be organized. The limit is extremely high and 
new methods of analyzing this information will emerge, they are indeed emerging already. Information on how these organisms are changing in different environments may provide critical inputs on how to test these methods and ideas. Space research may contribute to the solving of this challenge pushing forward how we understand biological processes.

So far we have defended the optimal nature of Drosophila melanogaster for this objective. Nevertheless, there may be also shortcomings in the decision of choosing this organism as any other organism that has become since many years ago the material of research in biological laboratories. The plasticity of biological systems may have produced a special type of organism. The standard laboratory wild type Drosophila is the product of a century of laboratory work (Kohler, 1994). They have become adapted to the relatively simple life style in them. They have been stripped out of their natural repository of variability. Selective inbreeding and additional manipulations could have suppressed much of the hidden genetic variability necessary for survival in the wild. Competition between different individuals derived from changing environmental factors has been minimized allowing the survival of less fit individuals. Therefore, are laboratory strains of Drosophila melanogaster the ones to be used in these experiments or should we turn to specimens caught directly from nature? Has not Drosophila melanogaster been following peasant populations originally from South Asia around the world? These are queries that can only hopefully be addressed by the types of experiments that the new Biology, based on Genomics and Proteomics, allows us to perform nowadays. The advantages of the huge information available on this organism makes difficult to escape from the conclusion that it should be one of the main actors of the new phase of Space exploration, but is it really the best one?

\section{The experimental approach with Drosophila.}

1) In vivo observation of Drosophila development using specific probes adapted to the detection of different developmental processes, namely, cell proliferation, cytoskeleton (CSK) and gene expression patterns including signal transduction pathways.

Embryonic development is usually very robust and apparently independent of most environmental conditions. As we have mentioned, this robustness may be responsible of the normal development in Microgravity of wild type embryos. One approach in ground control experiments is to find out whether transient developmental alterations caused by simulated Microgravity or Hypergravity treatments may be more readily seen or enhanced in appropriate hypomorphic mutants in one of the processes known to be altered in cells cultured in Microgravity, such as the cytoskeleton organization, the signal transduction pathways or the cell proliferation patterns.

Two types of observations are foreseen: The main one is the dynamic in vivo observations using appropriated markers. This will be complemented by the postexperimental analysis of fixed samples. In the ISS, it will be possible to monitor the invivo behavior of the fluorescent markers in Microgravity using one of the ISS microscopes equipped with fluorescent illumination. Moreover, in the ground simulation 
experiments, this dynamic observation will be also possible and the experiments will involve the immediate observation of embryos exposed during different periods of time to the simulation conditions. In these experiments the particular expression patterns of certain developmental regulatory and selector genes that are under the control of cascades of similar genes expressed in the same cell or in near-by cells will be studied.

The current power of the molecular methodology has made possible to visualize in vivo the behavior and switching on/off of defined genes. The method employs chimeric DNA molecules where the relevant sequences of these marker genes are coupled to a socalled reporter gene. The more popular one is the green fluorescent protein (GFP) from the cnidarian Aequorea victoria which is capable of producing fluorescence without an exogenously added substrate (Amsterdam et al, 1995). It can be linked into chimeric genes which have been visualized in vivo in Drosophila embryos, larvae and adults (Yeh et al, 1995, Barthmaier and Fyrberg, 1995). This can be done directly, coupling the reporter gene to the actual promoter of interest or indirectly, for instance, through the use of a specific activator systems, for example, one derived from yeast, the Gal-4 system. Thus, the many Gal-4 enhancer trap lines currently available in Drosophila can be used to achieve this objective. In them, the Gal-4 activator is under the control of one of the many controlling sequences in Drosophila reproducing the patterns of the endogenous genes. This is achieved by crossing the flies which have these enhancer traps with transgenic flies in which the reporter gene is expressed under the transcriptional control of the upstream activating sequence recognized by Gal-4. In many cases, these flies are fully viable, providing embryos that will directly produce a fluorescent product marking a particular tissue or cell structure that can be monitored under a fluorescent microscope without further manipulation.

In this way, the main development of Drosophila can be followed by microscopic observation of live unstained embryos. As will be described below a whole set of fluorescent components, markers of different subcellular elements and processes are now available. Nuclei, cytoskeletal components, mitochondria, membrane receptors, etc. can be now expressed in defined tissues and/or moments of development, providing a simple method to observe dynamically how different embryonic processes actually proceed.

For example, even before gastrulation, the embryo is subdivided in zones: namely, a) Mitotic domains (Foe, 1989), b) the precursor zones of the segments, marked by a series of stripes of defined gene expression domains in the antero-posterior axis, and c) several control genes are activated in the dorso-ventral axis, marking the subdivision into mesoderm, neuroectoderm and epidermis. Many of these control genes are transcription factors, but others are cell signaling and/or signal transduction pathways components. Morphogens are synthesized and secreted by certain cells to interact with neighboring cells endowed with appropriate receptors to induce the activation of a particular different state, usually by a chain of signal transduction pathways. These inducing phenomena due to the interaction of neighboring territories is one of the earlier recognized phenomena participating and shaping the complexity of embryonic development.

The actual number of components whose cell expression can be monitored is very large. Information on the transcription regulation and signal transduction cascades is 
becoming readily available in Drosophila. The main question is to decide which ones to try or to focus on. As mentioned before, looking into the changes in overall gene expression patterns of adult flies in Microgravity using microarray techniques may help in this decision. Since the processes so far found altered in Microgravity, real or simulated, are cellular related events, implicated in the mechanisms of proliferation, signal transduction and/or cytoskeletal organization, we are going initially to chose markers of events occurring before and after blastoderm cellularization. Before blastoderm cellularization the whole embryo is a huge single cell territory where nuclei are moving and dividing. After cellularization cells are much more smaller. Therefore, these different stages provide very different targets to the alteration in the gravitation vector. The experiments performed in the Ground Simulation Facilities should be critical to clarify which ones of the many possible markers may be more informative to be used in Space.

As a first approximation, three processes seem the best candidates for in vivo observation: 1) Markers of general cellular components, which can be used to follow cell division, movement and shaping during development (nuclei, cytoskeleton and cell membranes seem reasonable first choices). 2) Markers of signal transduction pathways. As a first possibility, we propose to use a particular well-studied process such as the embryonic dorsal closure at the end of germ band retraction since the involvement of several signal transduction pathways has been well established in this process (Jacinto et al, 2002). If we turn to defined genes, three types of genes seem prime candidates for this Investigation: A) The group of pair-rule genes, that produce seven stripes immediately after cellularization: fushi-tarazu, even-skipped or hairy among the better known ones. B) The group of segment polarity genes, such as engrailed, wingless, hedgehog or patched, that produce fourteen stripes, one for each segment and where hedgehog, one of the better characterized intercellular signals is operative. C) Tissue specific gene markers, such as those involved in mesoderm, ectoderm or nervous system specification. In addition, it would be interesting to monitor the cytoskeleton in the cells (Fig. 3). This could be attempted with cytoskeletal-GFP chimeric genes that express proteins capable of polymerizing into a particular cytoskeletal structure. The construction of such a hybrid with the Drosophila MER homolog (Moesin-like gene product) has been reported (Edwards et al, 1997). In this way it would be possible to monitor the Microfilaments Cytoskeletal Network in vivo. The production of a hybrid with the Nonclaret disjunctional (Ncd) microtubule motor protein fused to the green fluorescent protein (GFP) is capable of monitoring the microtubules (Endow, 1999). The incorporation of a nuclear signalling sequence in the GFP product labels the nuclei, etc. The selection of the more appropiate markers and the developmental processes to be followed will be done during the ground work in gravity simulation facilities. During this phase of the work, a hypomorphic mutant affecting dorsal closure will also be used, mutant selected with the help of Dr. Enrique Martín-Blanco (Centro de Investigación y Desarrollo, CSIC, Barcelona, Spain), one of the main investigators studying this process. An important point to make is that the GFP protein maintains its fluorescence in fixed material, what makes possible to combine in vivo on flight/simulation observations with post-experiment analysis after recovery. 
As mentioned, these developmental experiments can be complemented in the future with similar experiments using selected Drosophila strains, such as strains selected on the Ground for a different response in the developmental process found to be modified in Ground Gravity Simulation conditions. The increasing availability in Drosophila of a whole range of mutations that may reproduce or affect the same developmental process is well known. In this way, the possible mechanism of the Gravity environment on Development may be further investigated.

2) Aging and behavior in Gravity altered conditions.

Observation of Drosophila behaviour and aging in Ground Simulation Facilities. The methodology to be used is straightforward. The life span of the groups of flies will be followed by counting the number of flies that remain alive after different periods of the experiment. For hypergravity (centrifuge) and the Random Position Machine (3D clinostat) the Drosophila chambers developed for the ISS can be mounted and/or adapted to the Facilities. These chambers are provided with a transparent wall, facilitating the use of video observation for establishing the number of dead flies at different times during the experiment, as well as the level of motility. This parameter can be studied using the same observation capability and also using the infrared beam crossing incorporated in these containers.

The accommodation in the Magnetic Levitation Facility will be different. The flies will be incorporated in small cylinders with transparent walls. The actual size of the cylinders will have to be decided, since only the ones with tiny dimensions guarantee homogeneous levitation. The actual number of flies in the containers depends on their size, ranging from 5 flies in the smaller ones to 100 in the longer ones. Flies will probably not be able to feed while levitating in the Facility. For short-term experiments, of up to a few hours, this does not affect too much the survival of flies, but for long-term experiments, the experiment design will have to be modified substituting continuous levitation by periodic levitation, for instance, of a few hours a day. In any case, it is not clear whether long-term experiments will be possible in the Magnetic Levitation Facility. Effects on development will be examined by exposing embryos, larvae and/or pupae to this condition.

The experiment will be complemented by the fixation of some flies at different periods. In these fixed flies the level of defined gene expression, such as mitochondrial ones or even the overall pattern detected using microarray techniques can be followed after recovery from the facilities.

As done before, experiments will be performed with males to avoid the complication of contamination of the containers with embryos and larvae laid by the females. Nevertheless, in future Space experiments incorporating flies from successive generations in Space, they will include mixed populations of females and males. This is a consequence of the difficulty of separating males from females in Space. To test mixed female and male populations in aging and behavior experiments, we will use a different feeding medium, the so-called sugar medium made up of only sugar and agar, a medium compatible with the survival of Drosophila adults but not with the survival of the larvae. 
In this sugar-medium it is possible to maintain alive, mixed female and male populations without the complication of the progeny growth completely restricted in these conditions.

Finally, the aging experiments can be complemented with oxygen consumption measurements at defined times of the life-span curve, using an oxygen consumption measurement unit (see below for discussing the problems in this measurement).

As already mentioned, these behavior and aging experiments can be complemented with similar experiments using specific Drosophila strains, such as strains selected on the Ground for a different response to the gravity vector or strains showing different longevity curves. The analysis of these experiments will be critical in the decision on which strain to include in Flight Experiments with higher likelihood of giving important information on the effects of the Space environment on living beings.

Post-experimental analysis. In addition to analyze in detail some of the parameters of the experiment (video-recordings and oxygen consumption rates, food consumption rates, morphology of the preserved flies, life-spans, etc.), fixed material will be recovered both from Ground Simulation experiments and from real Microgravity ISS flights. Immunocytochemical and fluorescent observation on fixed material will be possible. Several molecular parameters can be also measured in this material such as the levels of mitochondrial RNAs and/or defined proteins such as muscle proteins. As already mentioned, it has been reported that in vertebrates, muscle proteins gel electrophoresis profiles change as a consequence of the lower weight load in Microgravity (Kraemer et al, 2000). In addition, microarray analysis will establish the overall gene expression profiles in these aging populations of flies exposed to a gravity altered environment on the Ground and in Space. All this information will help and provide support in the identification of the altered mechanisms and processes.

\section{The Problems to be solved.}

\section{Constraints in the ISS.}

At least during the next few years, the people involved in Biological Space Research should learn to live with the limitations currently imposed in the utilization of the ISS. While the crew remains restricted to three austronauts, the amount of work performed by the crew outside the normal maintenance of the ISS will be very limited. Developmental experiments will require the replacement of feeding trays and in addition, trays will have to be manipulated to prepare embryos for in vivo observation in flight and for fixation, once every couple of weeks. For aging experiments, the flies require less attention, making the experiment almost crew independent. Adult flies will have to be fixed at certain moments during the experiment to test the level of nuclear and mitochondrial gene expression. Obviously, these constraints do not affect the simulation facilities that will allow a better definition of the Space experiments. The type of hardware we are building (see below) is able to maintain flies during long periods of time 
with little or no crew involvement. Automatic embryo and adult flies preparation and fixation units are being designed and constructed as well.

A second problem will be the limitation in Storage capabilities, especially deep freeze facilities, if the experiments are to be maintained during the whole period of time between refurbishment flights to the ISS (at least three months). Although a bit unclear, storage space certainly will be a scarce resource, a constraint that will emerge as soon as more experiments find their way into the ISS. Again, this problem is less important for ground simulation facilities. The optimization of the experiments in the Ground Simulation Facilities will be critical to maintain this requirement under control, since the experiments to be flown shall be fewer and better designed.

\section{How to grow and handle Drosophila in Space.}

The constraints already discussed make necessary the previous study and modification of current methodological protocols to make them compatible with the Space conditions. The necessary hardware for Drosophila growth and handling in Space has to be also designed, built and tested.

\section{Optimization and Adaptation of the fixation methodology of Drosophila embryos and adults to the Space conditions.}

As far as one can tell, few attempts have been made in the past of fixing and/or preserving in an inactive state in Space the organisms to be studied afterwards on the Ground. Drosophila embryos, larvae and imagoes are surrounded by tight envelopes and cuticles extremely difficult for fixating fluids to penetrate. In some cases, embryos were kept frozen, not an optimal preservation method for further study after recovery on the Ground (Marco et al 1996).

Even in the case of the work in ground laboratories, the preservation of living Drosophila animals is a not easily solved problem. Some success has been achieved in the case of Drosophila embryos but the methods are complex enough not to make easy their adaptation to the Space conditions (Steponkus et al, 1990, Mazur et al, 1992, Ashburner, 1992).

As we have been discussing, it is almost certain that Drosophila melanogaster, one of the key model systems in current Biological Research, will be among the organisms accommodated in the International Space Station (ISS) or in Future Space Missions connected with Life Sciences. For example, our Madrid group is interested in the study of the Drosophila long-term adaptation to this unusual environment. Thus, sooner or later, the problems of fixation of the different stages of development will have to be addressed. Experiments performed in the Madrid laboratory in the past couple of years have succeeded in adapting the current techniques to the Space constraints. Designing and building an automatized fixation unit is underway (see below). With some success, current protocols have been adapted to maintain fixed animals during at least three months, the minimum refurbishment shuttle flight time with minimal handling and maximal automation. These protocols can be used for immunocytochemistry, 
morphological examinations at the level of the Optical Microscope and Molecular Biology experiments involving DNA, RNA and possibly proteins.

\section{Simplified Permeabilisation/Fixation Protocols for Drosophila embryos.}

A permeabilization-fixation protocol, simple enough to be used in the Space conditions and able to preserve embryos during at least three months has been developed. The first step in the protocols, dechorionization by a short treatment with a hypochlorite solution, should not give many problems when carried out in the Space Conditions. On the other hand, the following steps in the classic protocols based on formaldehydeheptane treatments, are much more difficult to be adapted to Microgravity conditions. The much simpler Steponkus-Cadwell protocol (Steponkus et al, 1996) although capable to open holes in the embryonic vitelline membrane through which a good nuclear DAPI signal can be obtained, is far from optimal for immunocytochemistry.

The more successful protocol developed in the Madrid laboratory uses a commercial non-formaline fixative, NEOFIX ${ }^{\complement}$, during 10 min just after two short treatments with isopropanol and hexane (permeabilization reagent). After this step the embryos can be stored in methanol at $4^{\circ} \mathrm{C}$ and after sample recovery the procedure can be successfully completed with a post fixation step with Heptane/PBS and Heptane/Methanol. This protocol combines the first part of the Steponkus-Cadwell protocol and the last part of the Heptane classic protocol but without paraformaldehyde (PFA).

Using PFA as fixative, the methanol conservation of embryos can be extended more than a year without much loss of detail. The NEOFIX $($ P Protocol can be used in the ISS, and can detect the Central Nervous System at least after 16 weeks of conservation in methanol at $4^{\circ} \mathrm{C}$ (see Fig. 4). Preservation at room temperature causes significant losses of signal. Morphology is acceptable when studied at the optical microscopy level, but not surprisingly due to the use of alcohol based-fixatives, the preservation at the electron microscopical level is poor. One has to be reminded that getting good electron microscopical images from Drosophila embryos requires the utilization of strong fixation procedures using high concentrations of aldehydes and osmic tetroxide (Ashburner 1989), procedures difficult to adapt to the Space conditions and possibly incompatible with immunocytochemistry. Electron microscopical observation of Drosophila larval and imagoes tissues require dissection of the specimens and fixation of the isolated organs, again a procedure difficult to adapt to the Space conditions. In fact, if one examines the huge Drosophila literature, not many studies using electron microscopical approaches will be found.

\section{Immunocytochemistry and Molecular Biology Applications}

In addition to the ELAV antibody, specific for the Central Nervous System, antiTropomyosin (TM) \& anti-Troponin $\mathrm{T}$ (TnT) antibodies have been applied for immunodetection of the Muscular system in Drosophila embryos conserved in methanol for ten weeks at $4^{\circ} \mathrm{C}$. In the case of TM the immunosignal is weak using either PFA or NEOFIX $@$ fixation. In the case of the TnT antibody, the immunosignal is strong and 
similar in the two experimental conditions (data not shown). Thus, the success of long term-delayed immunocytochemistry is very much antibody dependent and the optimal conditions should be worked out in each case.

The NEOFIX $\subset$ protocol tends to return a weaker and more diffuse immunosignal than PFA fixation. These results suggested a less than optimal level of morphological preservation. The same embryo fixation protocols were followed by rehydratation and LR White Resin inclusion after 3,5 months in methanol. In Fig. 4B we can see a $2 \mu \mathrm{m}$ sections of a NEOFIX @ fixed embryo in phase-contrast microscopy. The embryo morphology was similarly preserved at the level of Optical Microscopy no matter the protocol used.

Apart from morphological and cell biological techniques, the studies were extended to Molecular Biology. The optimal and simpler preservation method for this purpose has been dehydration in cold anhydride acetone. Conservation at room temperature also gives acceptable results. In samples stored at $-20^{\circ} \mathrm{C}$ and $4^{\circ} \mathrm{C}$, conservation of rRNA is almost perfect during at least one month (data not shown). In Fig. $4 \mathrm{C}$ we show that rRNA is preserved after seven months in acetone at $4^{\circ} \mathrm{C}$ or even at room temperature (amount reduced approximately 2 fold). In RT-PCR experiments involving the detection of the Troponin $\mathrm{C}$ transcripts, all expected bands appear revealing the isoform pattern for embryos and for adults.

\section{Preservation of living embryos, larvae or imagoes of Drosophila. How to recover living samples of every generation?}

This is an open problem for Drosophila melanogaster. While some organisms, such as C. elegans, Artemia franciscana, even some other Drosophila and insect species have developed mechanisms of adaptation that allow the preservation of living animals or embryos during long periods of time with minimal requirements, so far this capability has not been solved in an uncomplicated way for Drosophila melanogaster. The methods that have been published which allow to preserve living embryos in frozen conditions are complicated and relatively inefficient and, thus, not easily applicable to the Space research. This fact has also the consequence of the maintenance of large collections of living stocks in the Stocks Centers and in the multitude of Drosophila laboratories around the world.

Drosophila embryos, larvae and imagoes are cold sensitive and will not survive relatively short exposures to low temperatures. The relatively cold sensitivity of Drosophila melanogaster (Czajka and Lee J, 1990, Steponkus et al, 1996) puts a limit to the possibility of using lower temperatures to increase the survival of defined generations until the ISS is refurbished, since the developmental embryonic, larval and pupation period can be slowed down only up to a month and a half (Petavy et al, 2001) by keeping the animals at $12{ }^{\circ} \mathrm{C}$, the minimum temperature compatible with relatively high survival numbers. On the other hand, preliminary experiments indicate that other Drosophila species, particularly, Drosophila subobscura has higher survival levels at lower temperatures. The genome sequencing of Drosophila subobscura is in a very advance stage and therefore it could provide an alternative if it were necessary. 
Obviously much more work has to be carried out before Drosophila methodologies are considered ready and adapted for long-term Space experimentation.

\section{Hardware situation.}

The Biolab facility is the laboratory designed to support biological experiments on micro-organisms, cells, tissue cultures, small plants and small invertebrates on board the ISS (see the chapter by Enno Brickmann). The Biolab will be installed inside the European Columbus module which is scheduled for launch in October 2004. In addition to its general facilities as the glovebox, incubators, or freezers, the Biolab uses standard Experiment Containers mounted on two centrifuge rotors that provide either Microgravity or variable accelerations. One is typically used as $1 \mathrm{~g}$ reference, allowing to study the specific effect of weightlessness. The experiment hardware located inside the experiment containers, the Experimental Support Equipment, are provided by the investigators. Ten types of Experimental Support Equipment have been tested by ESA as bread-boards for use inside the Experimental containers for experiments with cell and tissue cultures and with small animals (see the chapter by Enno Brickmann).

Under the sponsorship of the European Space Agency, we have designed, developed and tested the Experimental Support Equipment item1 (ESE-1) for Drosophila melanogaster, in accordance with the Experiment Container and the Biolab facilities requirements. For this purpose, the special constraints likely to be encountered in any ISS mission were taken into account, such as the scarcity in energy, storage room and crewtime availability. In addition, we have been taking into consideration that sample exchange and equipment refurbishment will take place not before than three months, when Shuttle Flights are expected to visit the Space Station. Consequently, any experiment in the ISS requires the development of specific methodologies and equipment. On the one hand, as already shown, we have modified basic handling techniques to make possible experiments with Drosophila in Space conditions. On the other hand, the hardware we have developed is capable of maintaining Drosophila multigeneration experiments in the ISS taking advantage of the Biolab/MSC facilities. In addition of this main unit we are designing devices for embryo preparation and observation in flight, fixation, preservation or storage. All the hardware presented here would give the opportunity to study the adaptation of Drosophila to space environment in terms of development and behavior, at the level of phenotypic, cellular and molecular analysis.

\section{Drosophila multigeneration and behavior studies with the ESE-1 main device}

The Experimental Support Equipment Item 1 (ESE-1) of ESA is an automated culture system for multigenerational experiments with insects. Based on a concept that maximizes automatization, ESE-1 will allow the culture in space of many generations of fruit flies to study adult behavior and embryo development with minimal participation of the crew. The main unit consists in two cultivation chambers and a set of six food trays that can be automatically transferred into the cultivation chamber at specific times to feed 
the flies. Photographs of an improved ESE-1 unit are shown in Fig. 5. The main improvements in the current device are the following: the chambers have been lined with a white Teflon covering, the windows are now made of glass instead of the old plastic ones. These changes allow a better visualization of the flies in the Chamber. Also, the drum has been tightened and the access from the trays to the chambers has been made more hermetic to avoid fly trapping and/or escaping.

Inside the unit chamber, an infrared light and detector can be used to automatically monitor fly activity in combination with the video images obtained through the transparent wall of chambers. To complete these observations, an analysis of the adaptation of the flies to normal gravity after a long-term space flight will be performed, in term of viability, geotaxic response, mating and fertility.

In ESE-1, the tray exchange may be programmed to take place during short periods of time (about 4 to 8 hours) to collect undeveloped embryos. The embryos and larvae in the food tray can have different fates: a) to be discarded and killed by the specific killing actuator, that provides enough heat to the trays to kill the embryos without affecting the stability of the feeding medium (see Fig. 5B), b) to be transferred to the second chamber to obtain a new fly generation, c) to be removed for in-flight observation with the Biolab microscope, and d) to be removed for fixation and storage until post flight analysis.

\section{A prototype of a Sample Collection/Fixation Unit}

As described, most fixation procedures require the use of different fluids and washing cycles between fluids. Furthermore, some of the fixation procedures may also be facilitated by certain treatments such as microwave enhancement (Medina et al, 1994) and mechanical operations, as filtrations or phase separations. As far as we know, no currently existing multi-user facility in the ISS allows for such a flexibility of experiment peculiar procedures, nor do they guarantee a reasonable safety level for the operations.

The approach suggested for biological sample fixation in an especially dedicated facility, consists of leaving the sample in the EC during the initial phase of sample preparation until the sample is confined within a container, which is suitable of being inserted into a common facility (this will be a requirement for the experiment developer). This facility shall be especially prepared for safe handling of hazardous fluids, and it shall be equipped with thermal control and with the possibility of microwave or UV fixation enhancement.

Figure 6 presents a basic block diagram for such a facility, which is basically a fluid handling facility. This facility could consist of two bodies. The first body would serve as storage for the different fluids, including waste fluids. Thermal control of the reservoirs does not need to be active, since this part of the facility can be stored in a cooler/incubator until use.

The second body would contain pumps and valves allowing fluids to circulate through the sample and physical stimuli to be applied to the sample. It may even be 
capable of taking a sample from the experiment container/culture chamber, and guiding the probe into a vial, which then undergoes the fixation or preservation treatment, or to an observation chamber capable of being mounted on an ISS microscope.

An example of a complex fixation process is tissue fixation for histological or cytological examination, or, alternatively, fixation of adhesive cells in culture. In particular, cultured cells must be detached from the surface on which they are growing before their fixation. This step requires removal of the culture medium, washing, enzymatic detachment (in most cases cells are trypsinized), and final wash of the loosened cells.

Exact amounts of chemicals and accurate time control are crucial parameters in maintaining the morphological quality of samples, as well as their molecular integrity, especially concerning the possibility of performing further investigations involving DNA.

A tentative experimental protocol could be as follows: The culture plate or the vial with the suspension of cells on micro-carrier is submitted to a continuous flow with a suitable buffer. A first flushing step replaces the old medium and washes the cells. A second sequence, in which the continuous flow is stopped, would allow exact trypsinization of the culture. Then the cells could be transported, through the second washing process, to a different chamber, in which they are stopped by a filter to be ready for the final treatment (reseeding, fixation, staining), in a similar way as described in the above example.

This device could also serve for Drosophila embryos permeabilisation, fixation and storage as described in a previous section. A modification of the concept will allow to store embryos and adults until post-flight for specific genetic expression analysis.

\section{The problem of the measurement of the oxygen consumption of the adult flies in Microgravity.}

Another problem to be solved is the availability of a procedure for efficiently measuring oxygen consumption of flies and/or other biological samples in Space. This is a problem that remains untackled. The critical problem is guaranteeing the hermeticity of the chambers where the measurements will be made and to avoid the influence of other organisms such as those that may grow in the Drosophila food. An adaptation of the adult fixation unit could work. Once the flies are transferred into a hermetically sealed unit, oxygen consumption can be measured. The basis for the measurement could be either the drop in the pressure as the carbon dioxide produced in respiration is fixed with a base such as is carried out in the old Warburg respirometer, the decrease in the $\mathrm{pH}$ of the $\mathrm{CO}_{2}$ fixating solution or any equivalent approach. If the unit could be mounted in the available centrifuges and in the Random Position Machines, control measurements at gravityaltered conditions would be possible. 


\section{The Future.}

\section{The next step. The long-term adaptation to the Altered Ground Simulation/ISS environment.}

Once the results of the current phase of research are known, the path will be open to start the preparation of a long-term goal, the establishment of a permanent colony of Drosophila in the ISS and the investigation of the changes that the organism may endure there.

In the case of these long-term experiments, if changes in developmental processes, in behavior and aging responses have been previously detected in Ground simulation experiments and in intermediate length experiments in Space, the study of how they will continue changing will be the focus of the experimentation. In these experiments, an important aspect will be the identification of the genes that may be changing as the experiment proceeds. In addition, the use of mutant strains may give clues on how to stop or even accelerate adaptations and changes. Isolating RNAs from the different generations after the recovery of fixed adult samples will allow to check the gene expression profiles using microarray technology: How many genes will change expression during the longterm adaptation to the Space environment? Will they be related to the possible phenotype changes that the animals are experiencing?

One problem with these experiments is the limited size of the experimental populations. Although large in comparison with other animal systems that may be also involved in Space Research, the actual size of the populations will be limited to about 50 individuals in each container, thus, probabilistic drift effects may play a big part in what we may detect. This is an argument to support that the experiment is carried out in parallel in several containers, probably at least four or six and that populations are occasionally mixed among the containers to increase the actual effective population size. Carrying these experiments well in advance in ground simulation conditions may help us in better designing the real Space Experiments. Actually the ESE-1 container has a special tray that will allow mixing of populations in the two chambers.

\section{What can we expect?}

In short or intermediate length experiments, namely, those in which one or several generations of Drosophila will be grown in Space, there are two extreme distinct possibilities with regard to development: A) Clear detectable differences may appear between the developmental mechanisms and processes in simulated and real Microgavity and those that take place under normal gravity conditions, independently of whether the overall process is finally regulated and the final outcome is a normal larva and fly. In this case, the use of hypomorphic mutants affected in the same processes probably will enhance the differences, making possible the identification of strains that are able to develop under normal gravity conditions but they may fail doing so in simulation and real Microgravity conditions. A possible complication is when the results of the ground simulation experiments also differ from the ones obtained in real Microgravity (in the 
ISS). If so, further experiments trying to establish the nature of the difference will be required. B) No detectable differences appear. In this case, the conclusion is that the involvement of the gravity vector in the current developmental processes is insignificant if any and therefore, unless long-term effects appear, development will not be an obstacle to the future plans of the involvement of complex animal systems in the future Space Exploration (but see below).

With regard to aging and behavior, there are again two extreme possibilities. The motility changes in the fly behavior may continue correlating with the aging effects. Longer exposures (up till two months) to simulation/ real Microgravity will increase the severity of the effects. Flies selected for delayed aging may show a different response than flies selected for short life spans. Mitochondrial genes and function are affected. Oxygen consumption in altered gravity is increased. The use of flies which have lost the gravity perception mechanisms by mutation and/or selection do not show the same response as the normal wild type flies. Alternatively, the effects may become less important as the exposure to the Microgravity conditions is increased.

In genotypic terms, how many genes may change in expression? The use of microarrays will show if the group of genes expressed in the different developmental stages and during the aging process in simulated and real Microgravity are different from the normal gravity conditions. Do different strains with different gravity perception and/or life-spans show differences in the repertoire of expressed genes?

Now we will turn to the possible phenotypic responses in long-term experiments, namely, in experiments where a permanent colony of Drosophila will be established in Space:

Loss of graviresponse and the abnormal motility. One possible outcome of the long-term adaptation of Drosophila melanogaster to the Space environment is a possible change in the abnormal motility response. It can be anticipated that the behavior of the different generations of Drosophila will become more normal. If that is the case, will also this occur in the Ground Simulation experiments? If the change happens only occasionally, will it be limited to certain groups of flies (will stochastic events be involved in the changes?) or will they happen more consistently? How will the patterns of gene expression change in these populations? Which will be the actual genetic, developmental and physiological mechanisms involved in these changes? Will the graviperception mechanisms, not well understood yet in Drosophila become altered in these animals? How will they behave when recovered and transferred back to Earth? How will the aging pattern of these animals become modified? These and other interesting questions could be addressed in these experiments.

Changes and adaptation of muscle/Motility. In mammals, changes in the isoform pattern of muscle proteins have been reported after short-term exposure to Microgravity, similar to the ones found in ground conditions where gravity effects are counteracted, just as bed laying and/or hindleg suspension in model systems. Similar information is missing in the case of invertebrates. Drosophila changes in motility behavior in Microgravity but no information on changes in isoform patterns in Drosophila muscle has been reported, 
although there is an increasing knowledge on the patterns of isoform types associated with the different types of musculature in the fruitfly. It is likely that some changes may be also occurring in insects. The possibility of correlating possible changes with the functional changes detected in the behavior of the fruitfly is an interesting outcome of this analysis.

Changes in flying/jumping capability. In previous Space experiments it has been noticed that fruit flies in Microgravity seldom attempt to jump, the first step in the initiation of flight. Flies cannot take off by directly flying but they jump first using a strong second leg muscle, called TDT (Tergite depressor of trochanter). In contrast, flies in the same type of containers on the Ground do attempt to jump much more often. This is an observable change in behavior that can also be studied in long-term experiments to monitor if there are changes in this kind of behavior as new generations grow in Microgravity. This means that flies probably are less prone to fly in this environment, something that has not been directly tested but it is suggested by the observations mentioned at the beginning of this paragraph.

Finally, changes in size and shapes of the fruit flies and/or some of their organs, as well as changes in the size and shapes of the embryos and on the developmental mechanisms affected by Real or Simulated Microgravity could take place after long term exposures to this abnormal environment. Both are relative simple type of observations easily monitored during these experiments. In the case something of this sort is detected, it may give ideas of how the changes may be occurring and which would be their selective advantage if any.

From a genetical point of view, several key questions may be addressed in these experiments, especially if the strains used in the Space/simulation experiments have been selected taking into account the properties of their genomes, the location of potential transposable elements, heterochromatin features, etc.

How stable is the eucaryotic genome in Space? The use of these strains will allow to detect possible long-term changes in overall chromosome architecture or more likely changes in the sites and numbers of transposable elements. It is perhaps not very likely to find many changes in these key elements of the cellular architecture of high organisms, but it may be important to confirm the stability of the genome in this new environment, as a first stage in any attempt to send other organisms, such as human beings, in long exploration or even colonization initiatives.

Are the changes due to selection, random drift or both? The identification of any change in the phenotypic/genotypic properties of Drosophila flies exposed during multiple generations to the Space environment, will open the way of analyzing if the changes are selected as a consequence of their adaptive nature or simply the consequence of chance (random drift). To explore this alternative, it will be necessary to run replicas of the experiments both simultaneously or successively. 
Are the changes reversible? Finally, the possibility of bringing back samples of the fruit flies to more normal environments could test the capability of reversion of the changes.

\section{The final step: Towards the colonization of another planet or celestial body. Life Support Systems.}

The problems discussed in this article have two main objectives: 1) to identify and estimate the importance of the genotypic and phenotypic changes that biological systems may endure if exposed for very long-periods of time to strange environments likely to be found during the next steps of the Space Exploration Program. In particular, if human beings participate in this enterprise, it is important to find out the possible effects that they may experience. In this respect, the use of model systems that may show the effects much faster than the relatively long-lived, slowly reproducing human species seems mandatory. 2) It is possible, or even likely, that sooner or later the scenario of a possible colonization of a near-by extraterrestrial planet or satellite may become an objective, or even the objective of Human Society. To clarify the issues raised in this article is a prerequisite before this enterprise can be initiated. There are other prerequisites as well, for example, to extend the exploration of the possible targets for a colonization effort, to obtain as much information as possible of any remains or footprints of living or pre-living organisms that may have existed on these extraterrestrial locations (Maurel and Zaccai, 2001).

Independently of the mechanism of development involved, it is clear that the living organisms thriving in any site of the Biosphere have properties that are extraordinarily well adapted to the environmental conditions that they encounter in these sites. For example, in the case of the mammals, it is obvious that the skeletal framework depends on the size and weight of the animal, something that can be clearly seen if we present the skeletons of two different organisms, such as a cat and an elephant at the same scale (Fig. 7). Similar differences are also implemented in the circulatory system, which shall be able to pump blood to and from all parts of the organism. No one should harbor any doubts that the differences in developmental characteristics driven by the differences in the genes of the two species are responsible of these differences and that environmental effects will play only marginal roles in shaping the differences in morphology and properties at least in the short and intermediate run. Longer-term experiments of development in Microgravity could verify this assertion. Cats and elephants should maintain in the short-term, similar morphologies as they show on the Ground, even if they develop in the absence of gravity. At most, they may show accentuated effects of the osteoporosis and the circulatory changes observed by Austronauts in their current experience in Microgravity. But, what will happen in the long term? Will they not adapt to the levels that a smaller gravity force will impose to them? Is not this what evolutionary forces have been producing on living organisms since they become establish in our Planet? It is true that these evolutionary changes are extremely slow and that they may be marginal in even experiments lasting many years. 
Nevertheless, the knowledge of the changes that living organisms of all characteristics, but especially multicellular ones, may be experiencing will give information on the likelihood that organisms taking part in an effort to colonize another celestial body could be changing in directions undesired by us. This knowledge may prevent that the results of the operation produces undesired consequences.

Another problem that we have almost not mentioned yet but which should certainly be solved before any colonization attempts are initiated is understanding how multi-component systems are able to interact and become self-sustaining. The efforts that Space Programs are putting on the issue of Life Support Systems reflect the importance of the problem. As far as information is available, current life support systems are relatively simple and the work carried out on them concentrates on the issue of achieving selfsustainability for long period of times. Before any colonization attempts are made, these systems should be complicated and include living organisms of a higher organization. Model systems of the type discussed here, should be eventually included in these systems. Although not always useful for the maintenance of Humans, systems incorporating additional model organisms, such as yeast, Drosophila, Artemia (a small crustacean), Amphibian, Avian and/or Mammalian model systems should be explored in a direction that eventually will define the minimal system that may be used in such a venture. The actual details of this Research Program is outside the expertise and objectives of the authors of this chapter, but it is obvious that the scientists working in the next generation of Life Support Systems in Space will need the information derived from experiments such as those that we have discussed here.

Acknowledgements. Miquel Pastor (NTE, Spain), Jutta Kramer (originally at Fokker Space, The Netherlands), and several different ESA/Shuttle Teams with whom we have collaborated in the past should be acknowledged for their involvement and support in our work during the past years.

\section{References:}

Agarwal, S. and Sohal, R. S. (1994) DNA oxidative damage and life expectancy in houseflies. Proc. Natl. Acad. Sci. USA 91, 12332-12335.

Albrecht-Bühler, G. (1990) In defense of "Nonmolecular" Cell Biology. Int. Rev. Cytol. 120, 191-241.

Amsterdam, A. Lin, S. and Hopkins-N (1995) The Aequorea victoria green fluorescent protein can be used as a reporter in live zebrafish embryos. Dev. Biol, 171, 123-9.

Arbeitman, M. N., Furlong E. E., Imam, F., Johnson, E., Null B. H., Baker B. S., Krasnow M. A., Scott M. P., Davis R. W., White K.P. (2002) Gene expression during the life cycle of Drosophila melanogaster. Science, 297, 2270-5.

Arthur, W. (2000) The origin of Animal Body Plans J. Wiley \& Sons, Chichester.

Ashburner, M. (1989) Drosophila. A laboratory Manual , Cold Spring Harbor Laboratory Press, p 206-207.

Ashburner, M (1992) Frosted flies. Science, 258, 1896-7.

Barthmaier, P. and Fyrberg, E. (1995) Monitoring development and pathology of Drosophila indirect flight muscles using green fluorescent protein Dev. Biol. 169, 770-4. 
Benguría, A., Grande, E. , de Juan, E. , Ugalde, C. , Miquel, J., Garesse, R. and Marco R. (1996) Microgravity Effects on Drosophila melanogaster behavior and aging. Implications of the IML-2 Experiment. J. Biotechnol. 47, 191- 202.

Berry, M. V. and Geim, A. K. (1997). Of flying frogs and levitation Eur. J. Phys 18, 307313.

Bikle, D. D., Harris, J., Halloran, B. P. and Morey-Holton, E. (1994) Altered skeletal pattern of gene expression in response to Space Flight: comparison to the response to hind limb elevation Am. J. Physiol 267, 822-7.

Brock, J. P. (2000) The evolution of Adaptive Systems Academic Press, London

Burger, E. H. and Klein-Nulend, J. Mechanotransduction in bone-role of the lacunocanalicular network. FASEB J. 1999, 13 Suppl. S101-12

Calleja, M., Peña, P., Ugalde, C., Ferreiro, C., Marco, R. and Garesse, R. (1993). Mitochondrial DNA remains intact during Drosophila aging, but the levels of mitochondrial transcripts are significantly reduced. J. Biol. Chem. 268, 18891-7.

Chapes, S. K., Morrison, D. R., Guikema, J. A., Lewis, M. L. and Spooner, B. S. (1994) Production and action of cytokines in space. Adv. Space Res. 14(8), 5-8.

Claassen, D. E. and Spooner, B. S. (1994) Impact of Altered Gravity on Aspects of Cell Biology. Int. Rev. Cytol.156, 301-373.

Clejan, S., O'Connor, K. and Rosensweig N (2001) Tri-dimensional prostate cell cultures in simulated microgravity and induced changes in lipid second messengers and signal transduction. J Cell Mol Med. 5, 60-73.

Cogoli, A. and Cogoli-Greuter, M. (1997) Activation and proliferation of lymphocytes and other mammalian cells in microgravity. Adv Space Biol Med 6, 33-79

Cogoli, A. (1993) The effect of hypogravity and hypergravity on cells of the immune system J. Leukocyte Biol. 54, 250-268.

Cogoli, A., Bechler, B., Cogoli-Greuter, M., Criswell, S. B., Joller, H., Jollber, P., Hunziger, E. and Müller, O. (1993) Mitogenic signal transduction in T lymphocytes in microgravity. J. Leukocyte Biol. 53, 569-575.

Cogoli-Greuter M., Meloni M. A., Sciola L., Spano A., Pippia P., Monaco G. and Cogoli A. (1996) Movements and interactions of leukocytes in microgravity J Biotechnol 47 (23), 279-87

Cooke J., Nowak M.A., Boerlijst M. and Maynard-Smith J. (1997) Evolutionary origins and maintenance of redundant gene expression during metazoan development. Trends in Genet. 13(9):360-4.

Czajka, M. C. and Lee, R. E. Jr. (1990) A rapid cold-hardening response protecting against cold shock injury in Drosophila melanogaster. J. exp. Biol. 148,245-254.

de Groot, R. P., Rijken, P. J., Boonstra, J., Verkleij, A. J., de Laat, S. W. and Kruijer, W. (1991) Nuclear responses to protein kinase C signal transduction are sensitive to gravity changes. Exp. Cell Res. 197, 87-90.

de Groot, R. P., Rijken, P. J., Den Hertog, J., Boonstra, J., Verkleij, A. J., de Laat, S. W. and Kruijer, W. (1990) Microgravity decreases c-fos induction and serum response element activity. J. Cell. Sci. 97, 33-38.

Dobzhansky, T., Levene, H. and Spassky, B. (1972) Effects of selection and migration on geotactic and phototactic behaviour of Drosophila. Proc R Soc Lond B Biol Sci. 180(58), 21-41. 
Duprat, A. M., Husson, D. and Gualandris-Parisot, L. (1998) Does gravity influence the early stages of the development of the nervous system in an amphibian? Brain Res Rev. 28 (1-2), 19-24

Edgerton, V. R. and Roy, R. R. (2000) Gravitational biology of the neuromotor systems: a perspective to the next era. J. Appl. Physiol. 89(3), 1224-31.

Edwards, K. A., Demsky, M., Montague, R. A., Weymouth, N. and Kiehart, D. P. (1997) GFP-moesin illuminates actin cytoskeleton dynamics in living tissue and demonstrates cell shape changes during morphogenesis in Drosophila. Dev. Biol. 191, 103-17

Endow, S. A. (1999) GFP fusions to a microtubule motor protein to visualize meiotic and mitotic spindle dynamics in Drosophila. Methods Cell Biol. 58, 153-63.

Finch, C. E., Tanzi, R. E. (1997) Genetics of aging. Science 278, 407-11

Foe, V. (1989) Mitotic domains reveal early commitment of cells in Drosophila embryos. Development 107, 1-22.

Gerhart, J. and Kirschner, M. (1997) Cells, Embryos and Evolution Blackwell Science.

Goodwin, B. C. (1990) The evolution of Generic forms In Organizational Constraints on the Dynamics of Evolution J Maynard Smith \& G. Vida (eds). Manchester University Press, Manchester. pp 107-17.

Grimm, D., Bauer, J., Kossmehl, P., Shakibaei, M., Schoberger, J., Pickenhahn, H., Schulze-Tanzil, G., Vetter, R., Eilles, C., Paul, M. and Cogoli, A. (2002) Simulated microgravity alters differentiation and increases apoptosis in human follicular thyroid carcinoma cells FASEB J. 16, 604-6.

Gruener, R. and Hoeger, G. (1990) Vector-free gravity disrupts synapse formation in cell culture Am. J. Physiol. 258, c489-c494.

Gruener, R., Roberts, R. and Reitstetter, R. (1994) Reduced receptor aggregation and altered cytoskeleton in cultured myocytes after space-flight Biol. Sci. Space, 8:79-93.

Hammond, T. G., Lewis, F. C., Goodwin, T. J., Linnehan, R. M., Wolf, D. A., Hire, K. P., Campbell, W. C., Benes, E., O'Reilly, K. C., Globus, R. K. and Kaysen J. H. (1999) Gene expression in space. Nat Med. 5, 359.

Hemmersbach, R., Volkmann, D., Hader, D. P. (1999) Graviorientation in protists and plants J. Plant Physiol. 154 (1), 1-15.

Hirsch, J. and Ksander, G. (1969) Studies in experimental behavior genetics. V. Negative geotaxis and further chromosome analyses in Drosophila melanogaster J. Comp. Physiol. Psychol. 67 (1), 118-22.

Horn, E, and Foller, W. (1998) Induction of a gravity-related response by a single receptor cell in an insect Naturwissenschaften. 85, 121-3.

Husson, D. (1998) Etude du rôle de la pesanteur dans la fécondation et l' embryogènese de l' amphibien urodele Pleurodeles waltl, Ph. D. Thesis, Toulouse.

Jacinto, A., Woolner, S, and Martin, P. Dynamic analysis of dorsal closure in Drosophila: from genetics to cell biology Dev Cell 2002 3, 9-19.

Johnson, F. B., Sinclair, D. A. and Guarente L. (1999) Molecular biology of aging Cell 96, 291-302.

Kalthoff, K. (1996) Analysis of Biological Development McGraw-Hill, New York.

Khaoustov, V. I,, Risin, D., Pellis, N. R. and Yoffe, B. (2001) Microarray analysis of genes differentially expressed in HepG2 cells cultured in simulated microgravity: preliminary report. In Vitro Cell Dev Biol Anim. 37, 84-8

Kohler, R. (1994) Lords of the flies University of Chicago Press, Chicago and London. 
Kraemer, W. J., Staron, R. S., Gordon, S. E., Volek, J. S., Koziris, L. P., Duncan, N. D., Nindl, B. C., Gomez, A. L., Marx, J. O., Fry. A. C. and Murray, J. D. (2000) The effects of 10 days of spaceflight on the shuttle Endeavor on predominantly fast-twitch muscles in the rat Histochem. Cell Biol. 114, 349-55.

Lewis, M. L., Cubano, L. A., Zhao, B., Dinh, H. K., Pabalan, J. G., Piepmeier, E. H. and Bowman, P. D. (2001) cDNA microarray reveals altered cytoskeletal gene expression in space-flown leukemic T lymphocytes (Jurkat). FASEB J. 15, 1783-5.

Lewis, M. L., Reynolds, J. L., Cubano, L. A., Hatton, J. P., Lawless, B. D. and Piepmeier E. H. (1998) Spaceflight alters microtubules and increases apoptosis in humanlymphocytes (Jurkat) FASEB J. 12, 1007-18.

Li, X. and Noll, M. (1994) Evolution of distinct developmental functions of three Drosophila genes by the acquisition of different cis-regulatory regions. Nature, 367, 83-7. Lints F. A. and Soliman M. H. (1988) Drosophila as a Model Organism for Ageing Studies. Blackie and Son Ltd, London.

Malacinski, G. M. (1990) An essay on redundancy within developmental processes. In Cytoplasmic Organization Systems, ed. G. M. Malacinski, pp 123-151, Mc Graw Hill, New York.

Marco R., C. Díaz , A. Benguría, J. Mateos, J. Mas and E. de Juan (1999) The role of gravity in the Evolutionary Emergence of Multicellular Complexity. The Effects of Microgravity on arthropod Development and Aging Adv. Space Res 23, 2075-2082.

Marco, R., Benguría, A., Sánchez, J., \& E. de Juan (1996) Effects of the Space Environment on Drosophila melanogaster Development. Implications of the IML-2 Experiment J. Biotechnol. 47, 179-190.

Marco, R., González-Jurado, J., Calleja, M., Garesse, R., Maroto, M., Ramirez, E., de Juan, E. and Miquel, J. (1992) Microgravity effects on Drosophila melanogaster development and aging: Comparative analysis of the results of the Fly Experiment in the Biokosmos 9 URSS biosatellite flight Adv. Space Res., 12, (1) 157- 166.

Marco, R., Vernós, I., González, J. and Calleja, M. (1986) Embryogenesis and aging of Drosophila melanogaster flown in the Space Shuttle. Naturwissenschaften, 73, 431-432.

Marthy, H. J. , Gasset, G., Tixador, R., Eche, B., Schatt, P., Dessommes, A., Marthy, U. and Bacchieri, R. (1998) Skeletogenesis in sea urchin larvae under modified gravity conditions. Adv. Space Res. 21(8-9):1151-4

Maurel, M. C. and Zaccai, G. (2001) Why biologists should support the exploration of Mars Bioessays 23, 977-8.

Mazur, P., Cole, K. W., Hall, J. W., Schreuders, P. D. and Mahowald, A. P. (1992) Cryobiological preservation of Drosophila embryos. Science, 258, 1932-5.

Medina, F. J., Cerdido, A., Maroto, M., Manzanares, M. and R.Marco (1994) Enhancement of the Immunochemical detection of antigens by microwave irradiation. Benefits and limitations studied in isolated plant nuclei and Drosophila embryos in toto", Histochemistry, 102, 45-50

Mesland, D.A. M. (1992) Mechanisms of Gravity Effects on Cells: Are there GravitySensitive Windows Adv. Space Biol. \& Med. 2, 234-276.

Miller, M. S. and T. S. Keller (2000) Effects of Short Duration Microgravity on Drosophila Melanogaster (Fruit Fly) Activity http://www.emba.uvm.edu/VSGC/rocket/ rocket.htm

Moody, S. A. and Golden, C. (2000) Developmental biology research in space: issues and directions in the era of the international space station. Dev. Biol. 228, 1-5. 
Miquel, J. (1984) Effects of Microgravity and Hypergravity on invertebrate Development" In NASA Developmental Workshop Arlington, NASA TM 86756, p 7 -16.

Miquel, J. and Fleming, J. (1984) A two-step hypothesis on the mechanism of in vitro cell aging: cell differentation followed by intrinsic mitochondrial mutagenesis Exp. Gerontol 19, 31-36.

Miquel, J. and Fleming, J. (1986) Theoretical and experimental support for an "oxygen radical-mitochondrial injury". Hypothesis of cell aging in: Johnson J. E. Jr et al. (Eds), Free Radicals, Aging and Degenerative Diseases, Alan R. Liss, New YorK pp. 51-74.

Miquel, J. and Philpott, D. E. (1978) Experiment K202-Effects on weightlessness on the genetics and aging process of Drosophila melanogaster, in Rosenzweig, S. N. and Souza, K. S. (Eds) Final Reports of U. S. Experiments flown on the Soviet Satellite Cosmos NASA TM-78526, vol. 936, pp 32-59

Miquel, J. and Souza, K, A. (1991) Gravity Effects on Reproduction, Development and Aging Adv. Space Biol. \& Med. 1, 104-154.

Miquel, J., Economos, A. C., Fleming, J. and Johnson Jr, J. E. (1980) Mitochondrial role in cell aging Exp. Gerontol. 15, 575-591.

Miquel, J., Lundgren, P. R. and Binnard, R. (1972) Negative geotaxis and mating behavior in control and gamma-irradiated Drosophila Drosophila Inf. Service 48, 60.

Miquel, J., Lundgren, P. R., Bensch, K. G. and Atlan, H. (1976) Effects of temperature on the life-span, vitality and fine structure of Drosophila melanogaster Mech. Ageing Dev. 5, 347-70.

Moore, D. and Cogoli, A. (1996) Gravitational and space biology at the cellular level In Biological and Medical Research in Space (D. Moore, P. Bie and H. Oser, editors) p 1116, Springer Verlag.

Newman, S. A. and Comper, W. D. (1990) Generic physical mechanisms of morphogenesis and pattern formation Development 110, 1-18.

Orr, W. C. and Sohal, R. S.(1994) Extension of life-span by overexpression of superoxide dismutase and catalase in Drosophila melanogaster. Science 263, 1128-1130.

Partridge, L. and Barton, N. H. (1993) Optimality, mutation and the evolution of aging. Nature 362, 305-311.

Pearl, R. (1928) The rate of living London University Press, London.

Petavy, G., David, J. R., Gibert, P. and Moreteau, B. (2001) Viability and rate of development at different temperatures in Drosophila: a comparison of constant and alternating thermal regimes. J Therm. Biol., 26, 29-39

Pigliucci, M. (2001) Phenotypic plasticity. Beyond Nature and Nurture John Hopkins University Press, Baltimore and London.

Powell, J. R. (1997) Progress and Prospects in Evolutionary Biology. The Drosophila Model. Oxford Universiyt Press, New York.

Raff, R. A. (1996) The Shape of Life. Genes, Development and the Evolution of Animal Form, The University of Chicago Press, Chicago and London.

Reitstetter, R. (1994) Effects of vector-averaged gravity on single channel properties of the acetylcholine receptor in cultured myocytes NASA technical memorandum 94, 179.

Rose, M. R. (1991) Evolutionary Biology of Aging, Oxford University Press, New York.

Rose, M. R. \& Lauder G.V. (1996) Adaptation Academic Press, San Diego.

Schatten H, Lewis ML, Chakrabarti A. (2001) Spaceflight and clinorotation cause cytoskeleton and mitochondria changes and increases in apoptosis in cultured cells. Acta Astronaut. 49, 399- 418. 
Schatten H, Chakrabarti A, Taylor M, Crosser M, Mitchell K. (1998) The centriolecentrosome complex is affected by microgravity during cell division and in cilia of sea urchin embryos: results from space flight experiments. Microsc Microanal.4 Suppl 2:1132- 3.

Schmitt, D. A., Hatton, J. P., Emond, C., Chaput, D., Paris, H., Levade, T., Cazenave, JP., and Schaffar, L. (1996) The distribution of protein kinase C in human leukocytes is altered in Microgravity FASEB J 10, 1627-1634.

Schwarze, S. R., Weindruch, R. and Aiken JM (1998a) Decreased mitochondrial RNA levels without accumulation of mitochondrial DNA deletions in aging Drosophila melanogaster Mutat Res 382, 99-107

Schwarze, S. R., Weindruch, R. and Aiken, J. M. (1998b) Oxidative stress and aging reduce COX I RNA and cytochrome oxidase activity in Drosophila Free Radic Biol Med 25, $740-7$

Shigenaga, M. K., Hagen, T. M. and Ames, B. N. (1994) Oxidative damage an mitochondrial decay in aging. Proc. Natl. Acad. Sci. USA 91, 10771-10778.

Sohal R. S. and Weindruch, R. (1996) Oxidative stress, caloric restriction, and aging. Science 273 (5271), 59-63

Souza, K., A., Black, S. D. and Wassersug, R. J. (1995) Amphibian development in the virtual absence of gravity Proc. Natl. Acad. Sci. 92, 1975-1978.

Steponkus, P. L. , Myers, S. P., Lynch, D. V., Gardner, L., Bronshteyn, V., Leibo, S. P., Rall, W. F., Pitt, R. E., Lin, T. T., MacIntyre, R. J. (1990) Cryopreservation of Drosophila melanogaster embryos Nature 345, :170-2

Steponkus, P. L., Caldwell, S., Myers, S. P. and Cicero, M. (1996) Crypreservation of Drosophila melanogaster embryos Adv. in Low-Temperature Biology 3, 257-316.

Tautz, D. (2000) A genetic uncertainty problem Trends Genet 16(11):475-7,

Texada, D. J., Armstrong, J. D., Carter, E., Munjaal, R.P. and Beckingham K. M. (2002) Identification of genes involved in Gravitaxis in Drosophila 43d Annual Drosophila Research Conference San Diego Abstract 786.

Uva, B. M., Masini, M. A., Sturla, M., Prato, P., Passalacqua, M., Giuliani, M., Tagliafierro, G. and Strollo, F. (2002) Clinorotation-induced weightlessness influences the cytoskeleton of glial cells in culture Brain Res. 934, 132-9

Van Loon, J. J., Bervoets, D. J., Burger, E. H., Dieudonne, S. C., Hagen, J. W., Semeins, C. M., Doulabi, B. Z. and Veldhuijzen, J. P. (1995) Decreased mineralization and increased calcium release in isolated fetal mouse long bones under near weightlessness. $\mathrm{J}$ Bone Miner Res 10 (4), 550-7

Vernós, I., González- Jurado, J., Calleja, M. and Marco, R. (1989) Microgravity effects on the oogenesis and development of embryos of Drosophila melanogaster laid in the Spaceshuttle during the Biorack experiment Int. J. Develop. Biol. 33, 213- 226.

Vico, L., Lafage-Proust, M. H. and Alexandre, C. (1998) Effects of gravitational changes on the bone system in vitro and in vivo Bone 22 (5 Suppl):95S-100S.

Wallace, D. C. (1999) Mitochondrial diseases in man and mouse, Science 283, 1482-8.

Walther I, Pippia P., Meloni M. A., Turrini, F., Mannu, F. and Cogoli A. (1998) Simulated microgravity inhibits the genetic expression of interleukin-2 and its receptor in mitogenactivated T lymphocytes FEBS Lett. 436, 115-8.

Wang, E. (1999) Age-dependent atrophy and microgravity travel: what do they have in common? FASEB J. 13 Suppl, S167-74. 
Yeh, E., Gustafson, K. and Boulianne, G. L. (1995) Green fluorescent protein as a vital marker and reporter of gene expression in Drosophila Proc. Natl. Acad. Sci. USA, 92, 7036-40. 
Table 1.

Results of IML-2 Development Experiment

Drosophila larvae/flies developed from eggs laid by flies at the end of the third day of a Shuttle Flight of 18 days after the first embryo collection had been removed

\begin{tabular}{|l|c|c|c|c|c|}
\hline \multirow{2}{*}{$\begin{array}{l}\text { Number of developed } \\
\text { animals recovered }\end{array}$} & \multicolumn{2}{|c|}{ Flight Containers } & \multicolumn{2}{c|}{ Simultaneous Ground Control } & Trays* \\
\cline { 2 - 6 } & Static (0g) & Centrifuge (1g) & Static (1 g) & Centrifuge (1.4g) & \\
\hline Frozen & 2155 & 1875 & 1822 & 2028 & 7 \\
\hline Alive & 719 & 947 & 554 & 558 & 4 \\
\hline Total & 2874 & 2823 & 2376 & 2586 & 11 \\
\hline
\end{tabular}

* The number of trays recovered from the containers (two for each experimental condition) with 40 female and 10 male flies each 
Fig. 1 Results of Drosophila Motility video-recording in the IML-2 experiment. In the Figure, a reproduction of a snapshot (video image) from the experiment is presented including two images from two flight containers and one from the Ground experiment. The arrows in front of of the flies indicate which ones are moving during the short period of time following the recorded image. The size of the arrow measures the distance walked by the flies in 1/6 of a second afterwards. Summary numbers are given below. The fly enclosed in a dotted circle jumped during the recording of the image. While the flies in Microgravity do walk very actively, they rarely jump (this is the initial movement to initiate flying). 
Fig. 2 Scheme of potential links between the environment and the genotype-phenotype connection. 
Figure 3. Examples of Drosophila embryos labeled in the microtubular network (A in mitosis at an early syncytial blastoderm stage and B at a later embryonic developmental stage when complete cellularization has taken place) or in the microfilaments (C, the arrow points to the Central Nervous System. D. Microfilament involvement in dorsal closure, an embryonic operation dependent on cytoskeletal movements directed by signal transduction pathways (higher magnification). 
Fig.4. Different NEOFIXfixation results.
A. Immunocytochemistry with ELAV antibody
B. Phase-Contrast embryo microscopy
C. RNA extractions \&RT-PCR for Troponin cDNAs 
Fig. 5 Improved ESE 1 unit. A. Dismantled, the drum and the chambers can be seen as well as the trays loaded with feeding medium $\left(^{*}\right)$. B. Both chambers have been installed. View from behind with the killing actuator in the front (arrow). C. Complete loaded with flies. Right chamber door closed, left open with the medium visible. D. Close-up of the flies feeding on the tray. 
Figure 6: Possible concept for a fixation and preservation facility. Different fluids stored into a container specifically designed to store hazardous fluids are circulated in an experiment-defined sequence through the sample. The facility has the capability of transferring samples or part of the samples from one sample container to other (carried by circulating fluids), performing filtration, concentration processes, etc. and also has thermal control and possibility of electromagnetic radiation enhancement of the fixation activity. 
Fig 7. Skeletons of a cat and an elephant drawn at the same scale. It can be seen how these animals have adapted their morphology and organization of the same bone repository to meet the challenges of the Earth gravity. 


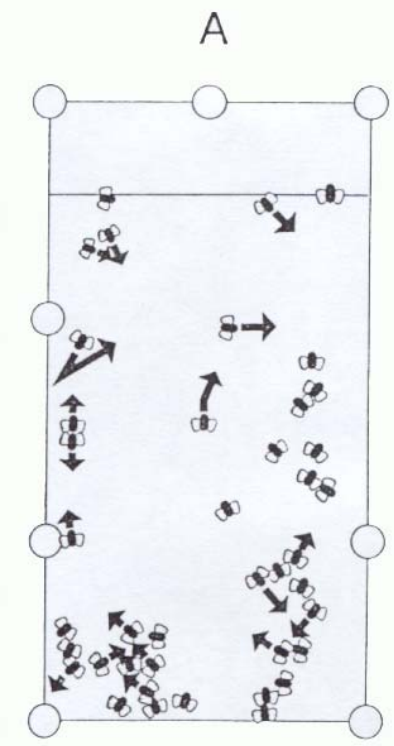

Flight

$40 \%$

Number of moving Flies

Average distance walked in $1 / 6$ of a second
B

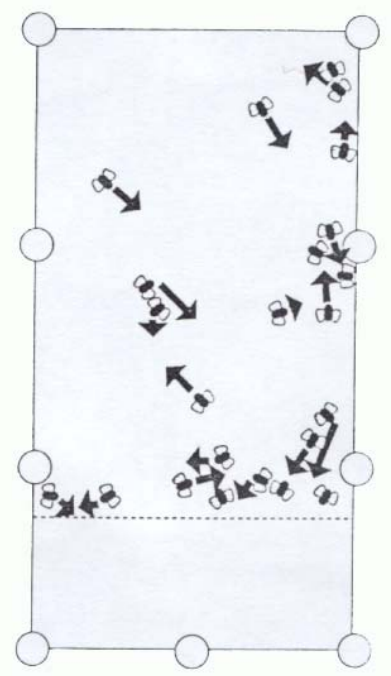

Flight

$60 \%$

$0.318 \pm 0.06$
C

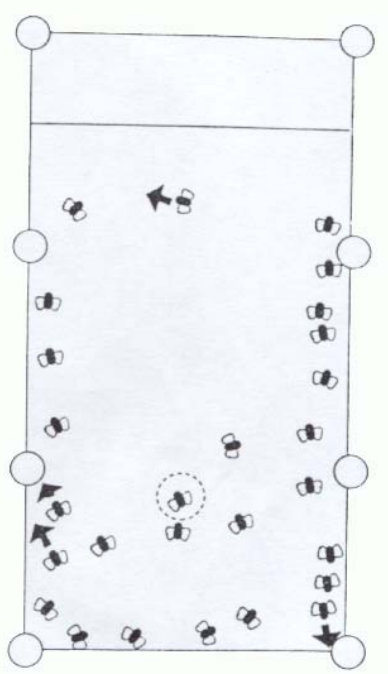

Ground

$0.007 \pm 0.002$ 


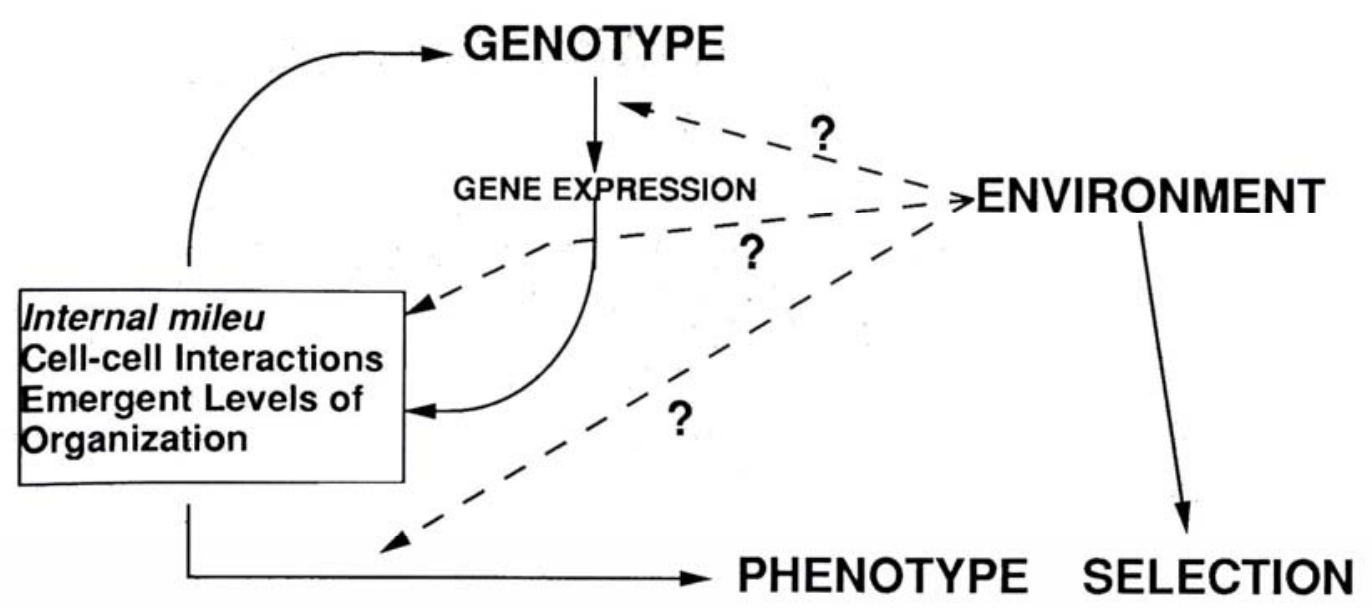



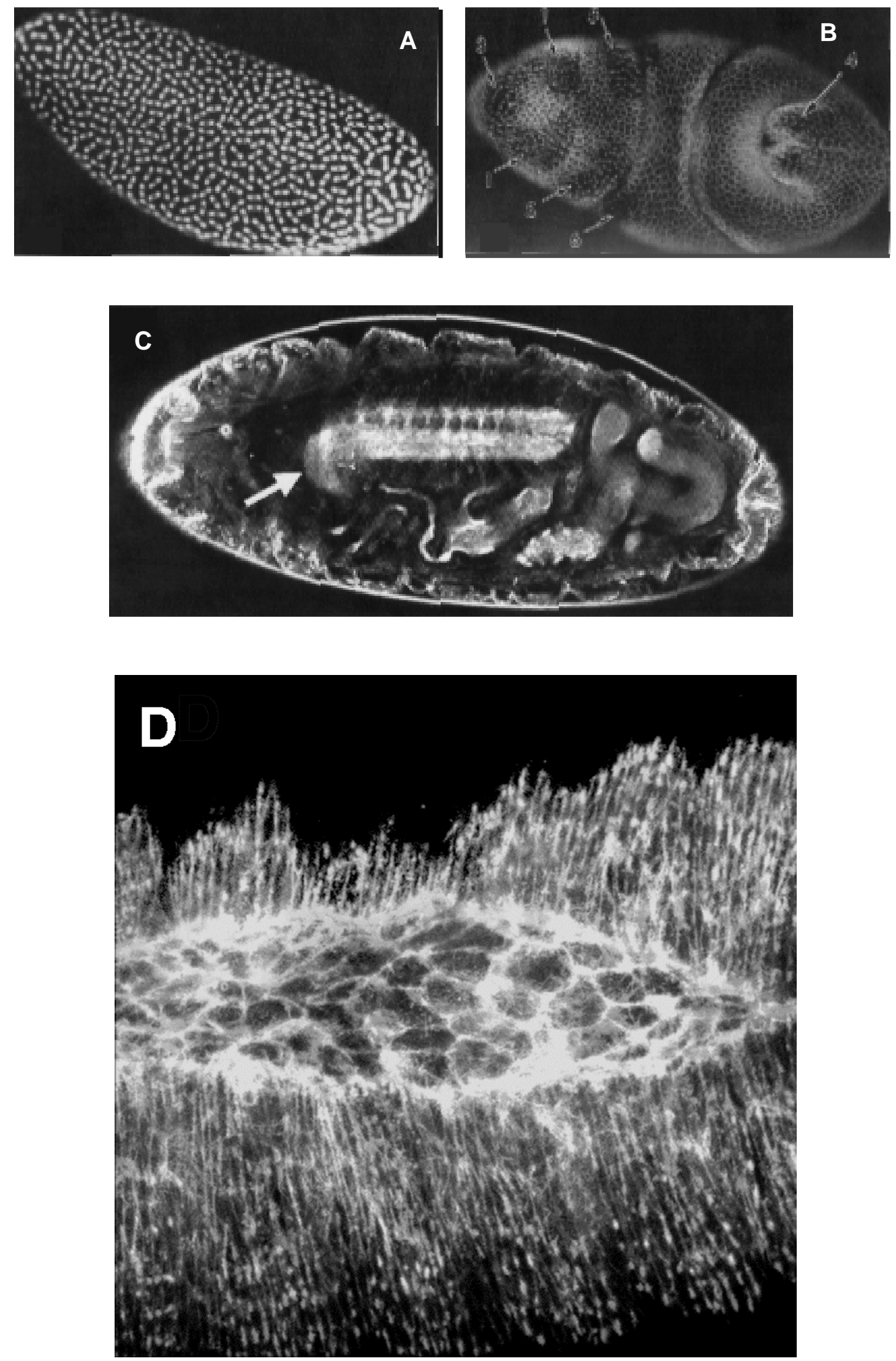


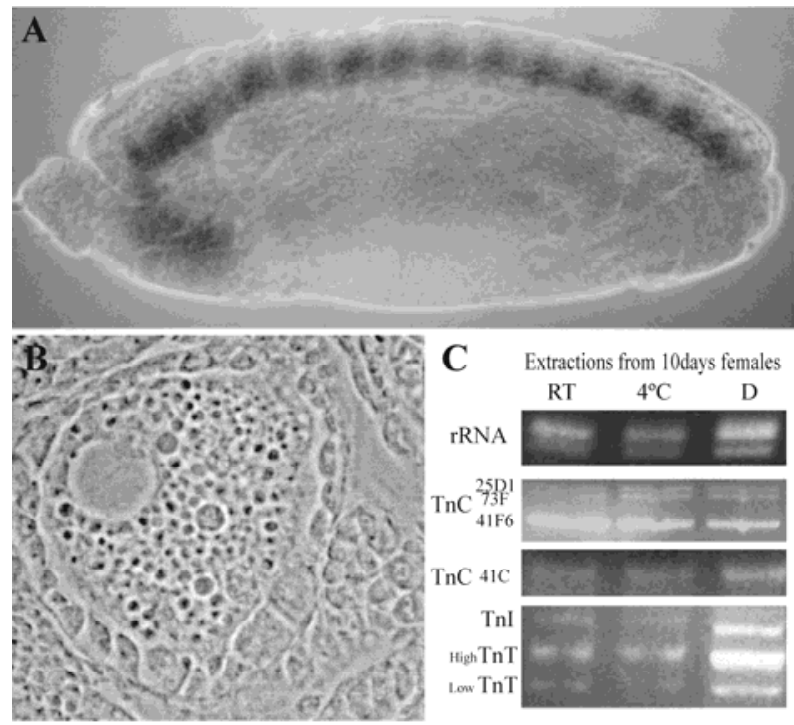




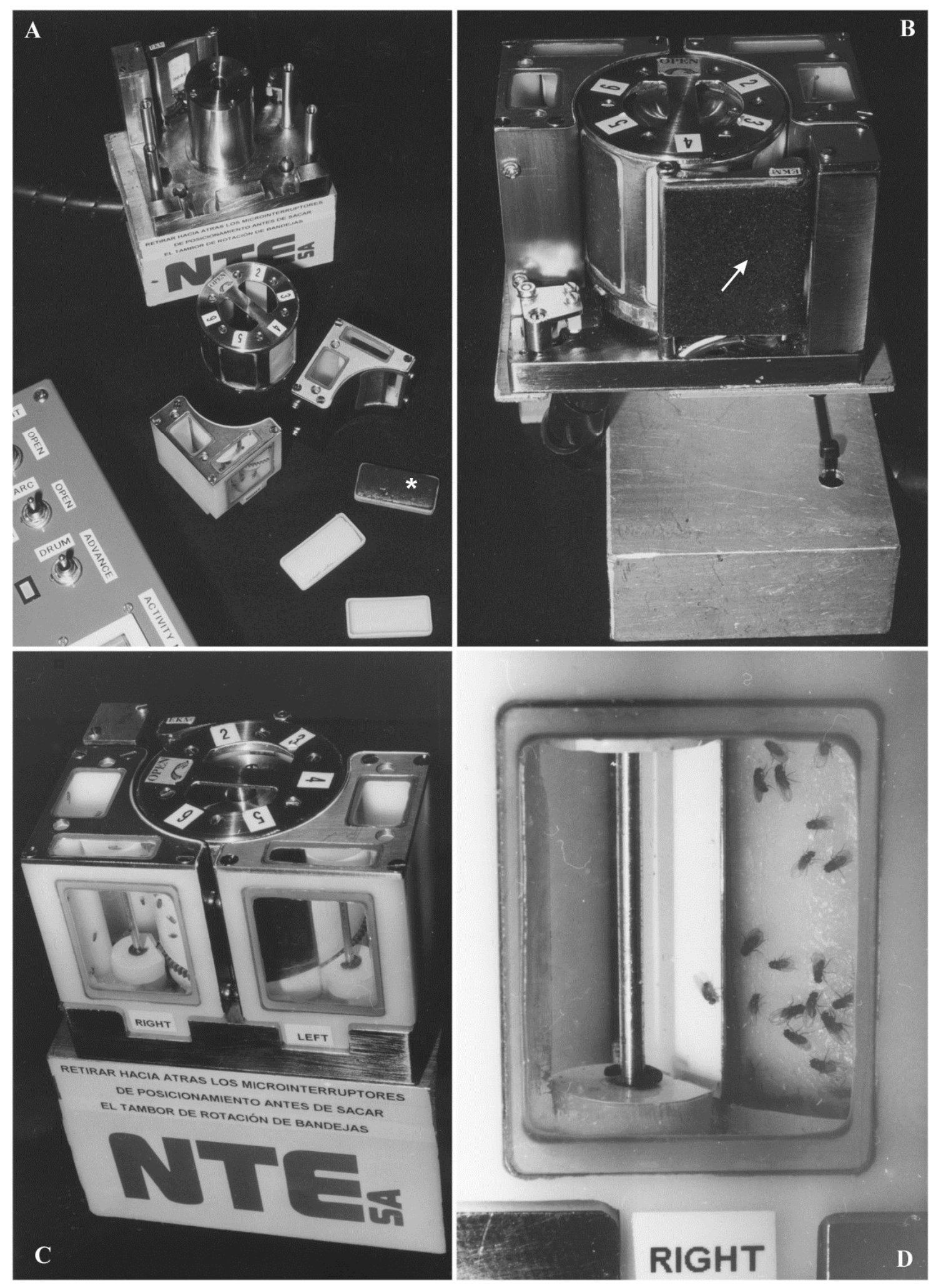




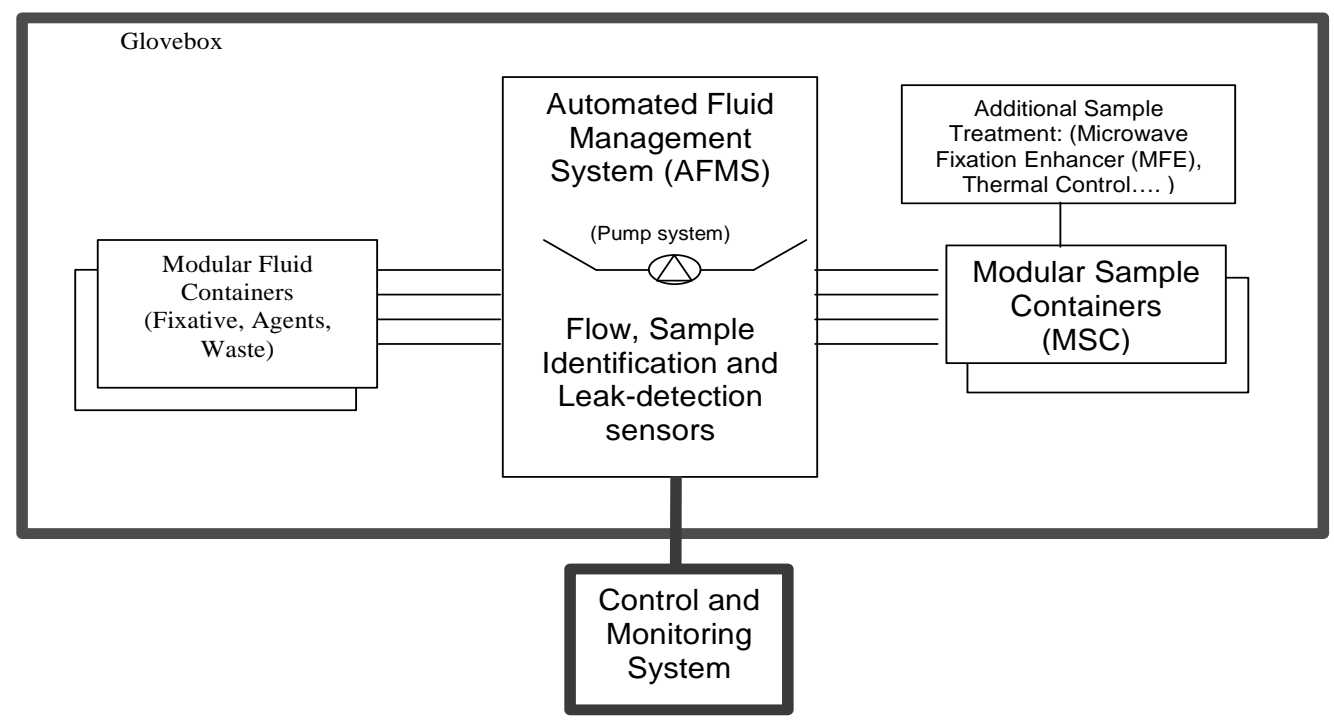




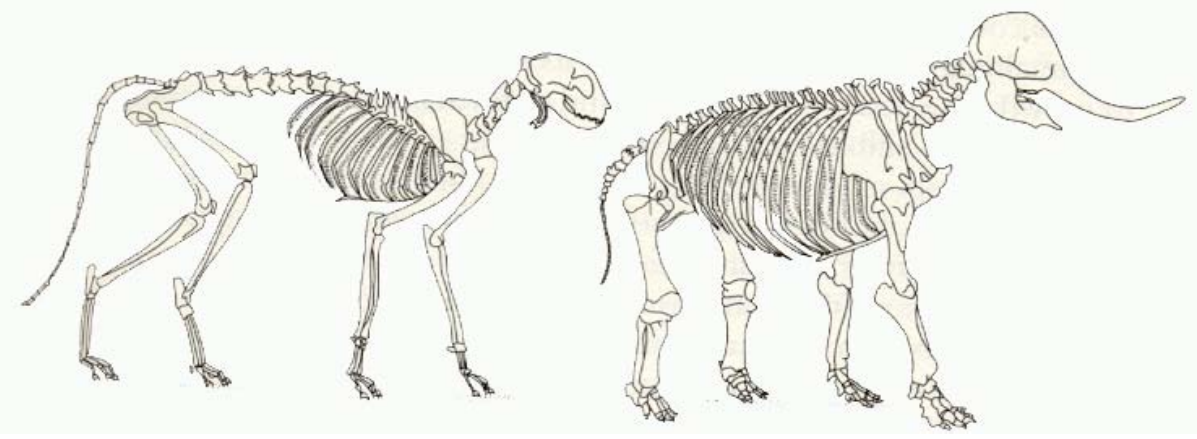


